KUNS-1621

hep-ph/9912496

\title{
Probing extra dimensions using Nambu-Goldstone bosons
}

\author{
Taichiro Kugof and Koichi Yoshiokal \\ Department of Physics, Kyoto University, Kyoto 606-8502, Japan
}

\begin{abstract}
We investigate a possibility that our four-dimensional world is a brane-like object embedded in a higher dimensional spacetime. In such a situation, the transverse coordinates of the brane become the Nambu-Goldstone bosons which appear as a result of spontaneous breaking of the translation symmetry. We determine the form of the effective action of the system, finding the explicit form of the vierbein induced on the brane in terms of the Nambu-Goldstone boson variables and the bulk vielbein. As was pointed out in the previous paper, the Kaluza-Klein mode couplings are suppressed by the effect of the brane fluctuation and the suppression is stronger if the brane tension is smaller. However, we here show that the brane tension cannot be arbitrarily small since the inverse of the brane tension gives the coupling constant of the Nambu-Goldstone bosons. A rather stringent bound is obtained for the brane tension and the fundamental ('string') scale from the consideration of the cooling process of the supernova.
\end{abstract}

\footnotetext{
*E-mail: kugo@gauge.scphys.kyoto-u.ac.jp

${ }^{\dagger}$ E-mail: yoshioka@gauge.scphys.kyoto-u.ac.jp
} 


\section{Introduction}

To understand the existence of various hierarchical scales in nature is one of the most important problem in particle physics. Recently it was proposed in [1] that the existence of extra spacetime dimensions may solve the gauge hierarchy problem between the Planck and weak scales in four dimensions. Stimulated by their work, there have appeared a large number of papers concerning the physics of extra dimensions. They deal with various phenomenological problems, such as fermion mass hierarchy [2], neutrino physics [3], supersymmetry breaking [4], flavor problems [5], cosmology and astrophysics [6], and so on.

It is really an interesting possibility that our four-dimensional world may lie on a 'brane' like a D-brane, orientifold plane, domain wall, etc. embedded in larger dimensions [7]. What are then typical signatures for such a brane world? Since there cannot exist a rigid body in the relativistic theory, any type of brane must necessarily fluctuate. Therefore there are always scalar fields which stand for the coordinates of the brane in the transverse dimensions. These scalar fields are the Nambu-Goldstone (NG) bosons which appear as a result of spontaneous breaking of the translation symmetry by the presence of the brane. Then one important way to explore the possibility of brane world is to investigate the effects of this NG bosons.

In higher dimensional models where the gravity and/or gauge fields live in the bulk, there are infinite numbers of Kaluza-Klein (KK) gravitons and/or KK gauge bosons on the brane. They couple to the matters living on the brane (our world) and can give visible effects in future collider experiments and astrophysics. These effects have been intensively investigated so far in many articles [11. However, in calculating some processes with KK excitation modes, there was a puzzling problem. That is, in summing up infinitely many KK modes, a tree-level amplitude diverges when the number of extra dimensions is larger than or equal to 2 . In the previous work [8], we showed that the higher KK mode couplings to the fields confined on the brane are exponentially suppressed if the effect of the above mentioned NG bosons is properly taken into account. That is, the suppression is due to the fluctuation of the brane. The suppression factor works as a regulator in the summations of KK

mode contributions and cures the problematic divergences. We also discussed some important phenomenological consequences of this factor. There, we concluded that if the brane tension is very small, the KK mode contributions become completely invisible from our world. 
In this paper, we will consider the effects of other interactions of this NG field and then show that we cannot freely have a small value for the brane tension. For this purpose, we will examine two physical phenomena. One is a new long-range force mediated by the NG bosons and the other is the cooling process of neutron stars in the supernova explosions. Both results involve the brane tension parameter $f$ (the decay constant of the NG boson) in the form $1 / f^{8}$ and lead to sizable effects if the tension becomes very small. That is, when the brane tension becomes small the KK mode couplings are suppressed but instead the effects of the NG bosons can

be measured. Therefore, we do not miss the possibilities of finding signatures of the extra dimensions in near future.

To discuss the effects of the NG bosons, we need explicitly construct the effective action on the brane. The construction of the action is more or less similar to that in the string theory, and is straightforward in principle, as was done in Ref. [9]. Although the author of Ref. [9] has given a definition of the induced vielbein, he unfortunately gave only a perturbative procedure for finding its form in terms of the bulk gravity and the brane coordinates (NG fields). Since it seems to have appeared in no literature, we give an explicit expression for the induced vielbein in this paper. The details of the derivation of the explicit form, together with its relevance to the non-linear realization theory, are given in Appendix A.

This paper is organized as follows. In section 2, we give the effective action for the system of the $d$-dimensional brane embedded in a higher dimensional spacetime where the gravity and gauge fields live in the bulk and the matter fields only in the brane. The NG boson part of this action is discussed in some detail in section 3. Given those interactions in the flat background, we consider several phenomenological effects of the NG field in section 4. Comparing them with observations, we can obtain constraints for physical parameters of extra dimensions such as the brane tension stated above. Section 5 is devoted to summary of this paper. Appendixes B and $\mathrm{C}$ are added for the calculations of the fifth force potential and of the cross sections for the two processes relevant to the star cooling.

\section{Setup}

The brane action is basically well-known in string theory [10]. So we here explain the setup briefly which we use throughout this paper, and construct the action for the 
system, in particular, by presenting the explicit form of the induced vierbein.

We take the brane to be a $(d-1)$-brane whose world volume topology is $R^{d}$. It is embedded in the bulk spacetime of dimension $D(>d)$ with topology $R^{d} \times T^{D-d}$, where $T^{k}$ denotes a $k$-dimensional torus. The coordinates of the bulk spacetime are denoted $X^{M}$, and those of the brane world volume are denoted $x^{\mu}$. The indices of upper-case Roman letters from the middle, $M, N, \cdots$, run over all dimensions, $0,1, \cdots,(D-1)$ and the Greek letter indices $\mu, \nu, \cdots$, run only over the first $d$ dimensions, $0,1, \cdots,(d-$ 1 ), while the lower-case Roman letters $m, n, \cdots$ run over the rest $(D-d)$-dimensions, $d, \cdots,(D-1)$; namely, we write like $M=(\mu, m), N=(\nu, n), \cdots$. The local Lorentz indices are denoted similarly by the corresponding letters from the beginning of the alphabet, like $A=(\alpha, a), B=(\beta, b), \cdots$. Our notation is summarized in table 1 .

\begin{tabular}{c|c|c}
\hline & bulk spacetime & brane world volume \\
\hline coordinate & $X^{M}(M=0,1, \cdots, D-1)$ & $x^{\mu}(\mu=0,1, \cdots, d-1)$ \\
\hline
\end{tabular}

\begin{tabular}{l|c||c|c}
\hline & $0,1, \cdots,(D-1)$ & $0,1, \cdots,(d-1)$ & $d, d+1, \cdots,(D-1)$ \\
\hline curved indices & $M, N, \cdots$ & $\mu, \nu, \cdots$ & $m, n, \cdots$ \\
\hline local Lorentz indices & $A, B, \cdots$ & $\alpha, \beta, \cdots$ & $a, b, \cdots$ \\
\hline
\end{tabular}

Table 1: Summary of our notation.

The action of this system consists of two parts; the bulk part $S_{\text {bulk }}$ and the brane part $S_{\text {brane. }}$ The bulk part action takes the form

$$
S_{\text {bulk }}=\int d^{D} X \operatorname{det} E\left[-\Lambda+\frac{M^{D-2}}{2} R-\frac{1}{4} G^{M R} G^{N S} \operatorname{tr}\left(F_{M N} F_{R S}\right)+\cdots\right],
$$

where $\Lambda, M$ and $R$ are the cosmological constant, the $D$-dimensional fundamental scale, and the $D$-dimensional scalar curvature, respectively. We have shown only gravity and Yang-Mills terms explicitly, $E_{M}^{A}(X)$ is the vielbein and $A_{M}(X)$ the YangMills fields. The bulk metric $G_{M N}(X)$ is given by the vielbein as usual

$$
G_{M N}(X)=\eta_{A B} E_{M}^{A}(X) E_{N}^{B}(X)
$$

where $\eta_{A B}$ is the $D$-dimensional Minkowski metric: $\eta_{A B}=\operatorname{diag}(+1,-1, \cdots,-1)$. The inverse matrices of $G_{M N}$ and $E^{A}{ }_{M}$ are denoted $G^{M N}$ and $E^{M}{ }_{A}$, respectively.

Now, let $Y^{M}(x)$ denote a point in the bulk spacetime which the point $x$ on the brane occupies. Considering the distance between two points $x$ and $x+d x$ on the 
brane and the parallel transport of a charged field from $x$ to $x+d x$, we see that the following metric and gauge fields are induced on the brane from the bulk ones, $G_{M N}(X)$ and $A_{M}(X)$ :

$$
\begin{aligned}
g_{\mu \nu}(x) & =G_{M N}(Y(x)) \partial_{\mu} Y^{M} \partial_{\nu} Y^{N}, \\
a_{\mu}(x) & =A_{M}(Y(x)) \partial_{\mu} Y^{M} .
\end{aligned}
$$

Similarly, a vielbein $e^{\alpha}{ }_{\mu}(x)$ on the brane is also induced from the bulk one $E^{A}{ }_{M}(X)$. The definition of this induced vielbein is, however, a bit non-trivial problem as was discussed by Sundrum [9] and hence is explained in detail in Appendix A. We derive there an explicit form of the induced vielbein, which we need to discuss the phenomenological implications of the NG bosons. The explicit form is given by

$$
e^{\alpha}{ }_{\mu}(x)=\mathcal{E}_{\| \nu}^{\alpha}\left(1+\left(\mathcal{E}_{\|}^{\mathrm{T}} \eta \mathcal{E}_{\|}\right)^{-1}\left(\mathcal{E}_{\perp}^{\mathrm{T}} \eta \mathcal{E}_{\perp}\right)\right)^{1 / 2 \nu},
$$

where we have defined

$$
\left(\begin{array}{c}
\mathcal{E}_{\| \mu}^{a}(x) \\
\mathcal{E}_{\perp \mu}^{a}(x)
\end{array}\right) \equiv\left(\begin{array}{l}
E^{\alpha}{ }_{M}(Y(x)) \partial_{\mu} Y^{M} \\
E^{a}{ }_{M}(Y(x)) \partial_{\mu} Y^{M}
\end{array}\right)
$$

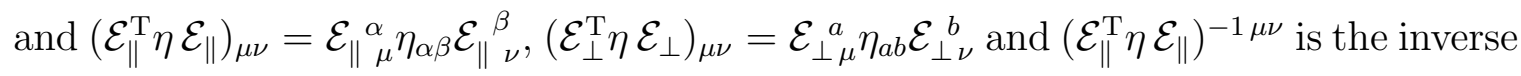
matrix of $\left(\mathcal{E}_{\|}^{\mathrm{T}} \eta \mathcal{E}_{\|}\right)_{\mu \nu}$. This vielbein satisfies the condition, $g_{\mu \nu}(x)=\eta_{\alpha \beta} e_{\mu}^{\alpha}(x) e_{\nu}^{\beta}(x)$ and gives a matrix connecting the local Lorentz basis to the curved one on the brane. The spin connection $\omega_{\mu}{ }^{\alpha}(x)$ induced on the brane from the bulk one $\Omega_{M}{ }_{B}{ }_{B}(X)$ is also given in Appendix A. It becomes the usual connection $\omega_{\mu}(e)$ given by the induced vielbein $e^{\alpha}{ }_{\mu}$ if the bulk connection is the usual one $\Omega_{M}(E)$ by $E^{A}{ }_{M}$.

The fields living on the brane generally couple to the bulk fields through these induced vielbein (or metric) and Yang-Mills fields. Let $\psi(x)$ be a fermion field on the brane which is charged under the Yang-Mills gauge group. Then, the brane part action takes the form

$$
S_{\text {brane }}=\int d^{d} x \operatorname{det} e\left[-\tau+e_{\alpha}^{\mu}(x) \bar{\psi}(x) i \gamma^{\alpha}\left(\frac{\overleftrightarrow{\nabla}_{\mu}}{2}-i g a_{\mu}(x)\right) \psi(x)-m \bar{\psi}(x) \psi(x)+\cdots\right],
$$

where $\bar{\psi} \overleftrightarrow{\nabla}_{\mu} \psi \equiv \bar{\psi} \nabla_{\mu} \psi-\left(\nabla_{\mu} \bar{\psi}\right) \psi$ with $\nabla_{\mu} \psi=\left(\partial_{\mu}+\frac{i}{4} \omega_{\mu}^{\alpha \beta}(x) \sigma_{\alpha \beta}\right) \psi$. The first term is a cosmological constant term on the brane with $\tau$ standing for the brane tension, and it also gives the Nambu-Goto action determining the motion (fluctuation) of the brane. The ellipsis contains other bosonic part terms for scalars and gauge fields if there exist such fields living only on the brane. These terms can be easily written by using the 
induced metric $g_{\mu \nu}(x)$. It should be noted that the total system with the action $S=$ $S_{\text {bulk }}+S_{\text {brane }}$ still keeps the gauge invariance under the bulk general coordinate, local Lorentz and Yang-Mills gauge transformations. Under the bulk general coordinate transformation, the induced fields $e_{\alpha}^{\mu}(x), a_{\mu}(x)$ as well as the genuine field $\psi(x)$ on the brane, all transform as scalar fields. This can be checked easily from the equations (2.3) -(2.5) if we note that $\partial_{\mu} Y^{M}(x)$ transforms as bulk vectors. The bulk local Lorentz transformation results in an $S O(1, d-1)$ local Lorentz transformation on the induced vielbein $e_{\mu}^{\alpha}(x)$ on the brane (see Appendix A), but $S_{\text {brane }}$ is manifestly invariant under any $S O(1, d-1)$ local Lorentz transformation. Moreover, under the bulk Yang-Mills transformation $A_{M}^{\prime}(X)=U(X) A_{M}(X) U^{-1}(X)+(i / g) U(X) \partial_{M} U^{-1}(X)$, the induced gauge field $a_{\mu}(x)$ is transformed as

$$
a_{\mu}^{\prime}(x)=U(Y(x)) a_{\mu}(x) U^{-1}(Y(x))+(i / g) U(Y(x)) \partial_{\mu} U^{-1}(Y(x)) .
$$

This is the usual gauge transformation in $d$-dimensions with $U(x)=U(Y(x))$, under which $S_{\text {brane }}$ is manifestly invariant.

The brane action $S_{\text {brane }}$ also has an invariance under reparametrization of the world volume coordinates $x^{\mu}$ as is familiar in string theory [10]. We fix this reparametrization invariance by choosing the gauge condition (the static gauge)

$$
Y^{M=\mu}(x)=x^{\mu} .
$$

Namely, we identify the world volume coordinates $x^{\mu}$ with the first $d$ components of the brane coordinate $Y^{M}(x)$ in the bulk. Then, the remaining $(D-d)$ components $Y^{M=m}(x)$ represent the brane coordinates transverse to the brane and behave as dynamical scalar fields on the brane. They are the NG fields appearing as a result of spontaneous breaking of the translation symmetries transverse to the brane.

If we take a suitable gauge fixing also for the local Lorentz invariance in the bulk, the induced vielbein can be written in a simpler form. Let us take the following local Lorentz gauge as is usually adopted in the case of dimensional reduction:

$$
\begin{aligned}
E_{M}^{A} & =\left(\begin{array}{cc}
E^{\alpha}{ }_{\mu} & E^{\alpha}{ }_{m}=0 \\
E^{a}{ }_{\mu} \equiv E^{a}{ }_{m} B_{\mu}^{m} & E^{a}{ }_{m}
\end{array}\right), \\
E_{A}^{M} & =\left(\begin{array}{cc}
E_{\alpha}^{\mu} & E^{\mu}{ }_{a}=0 \\
E_{\alpha}^{m}=-B_{\mu}^{m} E_{\alpha}^{\mu} & E^{m}{ }_{a}
\end{array}\right),
\end{aligned}
$$

which can be realized by using local Lorentz $S O(1, D-1) / S O(1, d-1)$ transformations. Note that $E_{\alpha}^{\mu}$ and $E_{a}^{m}$ are the inverse matrices of $E_{\mu}^{\alpha}$ and $E_{m}^{a}$, respectively. 
In this local Lorentz gauge (and in the static gauge), we have

$$
\begin{aligned}
\mathcal{E}_{\| \mu}^{\alpha}(x) & =E^{\alpha}{ }_{\mu}\left(x, Y^{m}(x)\right), \\
\mathcal{E}_{\perp \mu}^{a}(x) & =E^{a}{ }_{n}\left(x, Y^{m}(x)\right) \mathcal{B}_{\mu}^{n}(x), \\
\mathcal{B}_{\mu}^{n}(x) & \equiv B_{\mu}^{n}\left(x, Y^{m}(x)\right)+\partial_{\mu} Y^{n}(x),
\end{aligned}
$$

so that from (2.4) the induced vielbein becomes

$$
e^{\alpha}{ }_{\mu}(x)=E^{\alpha}{ }_{\nu}\left(\delta^{\nu}{ }_{\mu}+G^{\nu \rho} \mathcal{B}_{\rho}^{m} G_{m n} \mathcal{B}_{\mu}^{n}\right)^{1 / 2} .
$$

In the usual Kaluza-Klein gravity, the zero modes of the off-diagonal components of the higher dimensional metric, $B_{\mu}^{m}$, become massless gauge bosons of $U(1)^{D-d}$ in $d$ dimensions. They correspond to the gauged translational symmetry in the extra dimension. Now, since the translations are spontaneously broken by the existence of the brane, these gauge fields absorb the NG bosons $Y^{m}$ and become massive on the brane. In the above expression of the induced vielbein (2.14), we can see a part of this Higgs effect. That is, the gauge fields $B_{\mu}^{m}$ appear only in the form of $\mathcal{B}_{\mu}^{m}=B_{\mu}^{m}+\partial_{\mu} Y^{m}$ which correspond to the massive gauge fields.

It is interesting to note that even if the bulk gravity is absent, i.e.,

$$
E_{M}^{A}(X)=\delta_{M}^{A} \quad\left(G_{M N}(X)=\eta_{M N}\right),
$$

the induced vielbein $e^{\alpha}{ }_{\mu}(x)$ is non-trivial; $e^{\alpha}{ }_{\mu}(x) \neq \delta_{\mu}^{\alpha}$. In this case, the Eq. (2.14) takes a simpler form

$$
e^{\alpha}{ }_{\mu}(x)=\left(\delta^{\alpha}{ }_{\mu}-\frac{1}{\tau} \partial^{\alpha} \phi^{m}(x) \partial_{\mu} \phi^{m}(x)\right)^{1 / 2},
$$

where $\phi^{m}(x)$ are $(D-d) \mathrm{NG}$ scalar fields rescaled with the brane tension factor so that they carry the usual mass dimension $(d / 2-1)$ of scalar fields;

$$
\phi^{m}(x) \equiv \sqrt{\tau} Y^{m}(x) .
$$

Hereafter, we consider the flat background case, i.e., there is no object in the bulk except for the brane. We take into account the fluctuations of metric around this background. In this situation, the expression for $S_{\text {bulk }}$ after the torus compactification and the relevant phenomenology have been discussed in detail in Refs. [11]. In the following part of this paper, we will investigate the physics with the action $S=$ $S_{\text {bulk }}+S_{\text {brane }}$, focusing especially on the role of the NG field $\phi^{m}(x)$. 


\section{$3 \quad$ Effective action on the brane}

The fluctuation of brane is governed by the Nambu-Goto term in the brane action Eq. (2.6), which reads by inserting the form of the induced vierbein (2.16),

$$
\begin{array}{r}
\int d^{d} x \operatorname{det} e(-\tau)=\int d^{d} x\left[-\tau+\frac{1}{2} \partial^{\mu} \phi^{m}(x) \partial_{\mu} \phi^{m}(x)+\frac{1}{8 \tau}\left(\partial^{\mu} \phi^{m}(x) \partial_{\mu} \phi^{m}(x)\right)^{2}\right. \\
\left.-\frac{1}{4 \tau}\left(\partial^{\mu} \phi^{m}(x) \partial_{\nu} \phi^{m}(x)\right)\left(\partial^{\nu} \phi^{n}(x) \partial_{\mu} \phi^{n}(x)\right)+\cdots\right] .
\end{array}
$$

Note that $\phi$ has a properly normalized kinetic term by the rescaling $Y^{m}(x)=\phi^{m}(x) / \sqrt{\tau}$, and hence that all interaction terms of $\phi(x)$ are accompanied by some powers of $1 / \tau$. Noting also that the induced Yang-Mills field (2.3) now reads

$$
a_{\mu}(x)=A_{\mu}\left(x, \frac{\phi(x)}{\sqrt{\tau}}\right)+\frac{1}{\sqrt{\tau}} A_{m}\left(x, \frac{\phi(x)}{\sqrt{\tau}}\right) \partial_{\mu} \phi^{m}(x),
$$

the fermion part of the brane action is given by

$$
\begin{gathered}
S_{\text {fermion }}=\int d^{d} x \bar{\psi}\left(i \gamma^{\mu} \partial_{\mu}-m_{\psi}\right) \psi+S_{\mathrm{NG}}+S_{\text {grav }} \\
S_{\mathrm{NG}}=\int d^{d} x\left[g \bar{\psi} \gamma^{\mu} \psi A_{\mu}\left(x, \frac{\phi(x)}{\sqrt{\tau}}\right)+\frac{g}{\sqrt{\tau}} \bar{\psi} \gamma^{\mu} \psi A_{m}\left(x, \frac{\phi(x)}{\sqrt{\tau}}\right) \partial_{\mu} \phi^{m}(x)\right. \\
+\frac{1}{2 \tau}\left(\partial^{\mu} \phi^{m} \partial_{\nu} \phi^{m}-\delta_{\nu}^{\mu} \partial^{\rho} \phi^{m} \partial_{\rho} \phi^{m}\right)\left(\bar{\psi} i \gamma^{\nu} \frac{\stackrel{\leftrightarrow}{\partial}}{2} \psi+g \bar{\psi} \gamma^{\nu} \psi A_{\mu}\left(x, \frac{\phi(x)}{\sqrt{\tau}}\right)\right) \\
\left.+\frac{1}{2 \tau}\left(\partial^{\rho} \phi^{m} \partial_{\rho} \phi^{m}\right) m_{\psi} \bar{\psi} \psi+O\left(\tau^{-\frac{3}{2}}\right)\right] \\
S_{\text {grav }}=-\kappa \int d^{d} x h^{\mu \nu}\left(x, \frac{\phi(x)}{\sqrt{\tau}}\right) T_{\mu \nu},
\end{gathered}
$$

where $h_{\mu \nu}$ is $d$-dimensional part of the fluctuations of the vielbein, $E_{\mu}^{\alpha}=\delta_{\mu}^{\alpha}+\kappa h_{\mu}^{\alpha}$, and $\kappa$ denotes the $d$-dimensional gravity coupling constant which is related to the fundamental scale $M$ in (2.1) via $M^{D-2} V^{D-d}=\kappa^{-2} . V$ is the volume of the compactification manifold $T^{D-d}$ which is $(2 \pi R)^{D-d}$ here and when $d=4, \kappa$ is related to the Newton constant $G_{N}$ by $\kappa^{2}=8 \pi G_{N}$. The energy-momentum tensor $T_{\mu \nu}$ is given by

$$
T_{\mu \nu}=-\eta_{\mu \nu}\left(\frac{1}{2} \bar{\psi} i \gamma^{\rho} \stackrel{\leftrightarrow}{\partial}_{\rho} \psi-m_{\psi} \bar{\psi} \psi\right)+\frac{1}{2} \bar{\psi} i \gamma_{\nu} \stackrel{\leftrightarrow}{\partial}_{\mu} \psi+\frac{1}{2} \partial_{\rho}\left(\bar{\psi} i \gamma_{\mu} \sigma_{\nu}{ }^{\rho} \psi\right)
$$

assuming $h_{\mu \nu}$ symmetric. Here we have neglected the interaction terms between the fluctuations of bulk metric and the NG field, which are higher-order terms in the gravitational coupling constant and $\tau^{-1}$. 
From the above action, we can see that there are two types of coupling between the NG boson $\phi$ and the fields on the brane. One is the derivative couplings of $\phi$ (the second and third lines in (3.4) which come from the expansion of the induced vielbein (2.16). The derivative coupling is governed by $\tau$ which is the decay constant of this NG field. Another type of interaction originates from the fact that $\phi$ stand for the coordinates of brane in the transverse dimensions. To see the form of this type of coupling, we ignore all the derivative interaction terms of $\phi$ through $\partial_{\mu} \phi$. Then, the brane system is simply described by

$$
S_{\text {brane }}=\int d^{d} x\left[\frac{1}{2} \partial^{\mu} \phi^{m} \partial_{\mu} \phi^{m}+\bar{\psi}\left(i \gamma^{\mu} \partial_{\mu}-m_{\psi}\right) \psi+g \bar{\psi} \gamma^{\mu} \psi(x) A_{\mu}\left(x, \frac{\phi(x)}{\sqrt{\tau}}\right)\right]
$$

aside from the gravity coupling terms. We assume, for simplicity, that the extra dimensions are all compactified into a tori with a common radius $R$. Then the bulk gauge fields $A_{M}(X)$ is expanded into the KK modes labeled by a $(D-d)$ vector $n=\left(n^{m}\right)$ :

$$
A_{M}\left(X^{\mu}=x^{\mu}, X^{m}=Y^{m}\right)=\frac{1}{\sqrt{V}} \sum_{n} A_{M}^{(n)}(x) e^{i n \cdot Y / R} .
$$

Inserting the Kaluza-Klein mode expansion into $A_{\mu}$, the gauge interaction term reads

$$
\int d^{d} x \sum_{n} g \bar{\psi}(x) \gamma^{\mu} \psi(x) A_{\mu}^{(n)}(x) \exp \left(\frac{i n \cdot \phi(x)}{R \sqrt{\tau}}\right) .
$$

This interaction term apparently seems to imply equal couplings $g$ for all the KaluzaKlein excited modes. However, although $\phi=0$ classically it has fluctuations quantum mechanically, which is governed by the kinetic term $(1 / 2) \partial^{\mu} \phi^{m} \partial_{\mu} \phi^{m}$. We should rewrite the exponential factor into a normal ordered form

$$
\int d^{d} x \sum_{n} g e^{-\frac{1}{2} \frac{n^{2}}{R^{2} \tau} \Delta\left(M^{-1}\right)} \cdot \bar{\psi}(x) \gamma^{\mu} \psi(x) A_{\mu}^{(n)}(x): \exp \left(\frac{i n \cdot \phi(x)}{R \sqrt{\tau}}\right):,
$$

where $\Delta$ is the free propagator of $\phi$

$$
\Delta(x-y) \equiv\langle\phi(x) \phi(y)\rangle=\frac{-1}{4 \pi^{2}} \frac{1}{(x-y)^{2}} .
$$

In deriving this, a singularity at $x=y$ is cut off at the fundamental scale $M$ above which the effective theory description on the brane becomes invalid. The interaction term (3.10) implies that the effective coupling of the level $n$ KK modes to $d$ dimensional fields is suppressed exponentially. It should be noted that the same 
exponential factor also appears in the coupling with the KK gravitons $S_{\text {grav }}$ (3.5). In the following section, we consider the effects of the NG boson $\phi(x)$ with the derivative interaction terms in (3.4) and non-derivative one (3.10). We will see that these two interaction terms play complementary roles in obtaining the limits for the parameters of the model.

Here we comment on the coupling involving the bulk gauge filed $A_{m}$ in the action (3.4). When we perform a compactification from higher $D$-dimensional theories, the

KK modes $A_{\mu}^{(n)}$ for the bulk gauge fields get masses and so should absorb the physical degrees of freedom from some scalar fields. These are just supplied by the KK excitations $A_{m}^{(n)}$ of the extra-dimensional components; i.e., the induced gauge field (3.2) can be rewritten in the form

$$
\left.a_{\mu}(x)=\sum_{n \neq 0}\left[\widetilde{A}_{\mu}^{(n)}(x) e^{i \frac{n}{R} \frac{\phi(x)}{\sqrt{\tau}}}-i \frac{R}{n^{2}} \partial_{\mu}\left(n^{m} A_{m}^{(n)}(x) e^{i \frac{n}{R} \frac{\phi(x)}{\sqrt{\tau}}}\right)\right]+\text { (zero mode }\right),(3
$$

where $\widetilde{A}_{\mu}^{(n)} \equiv A_{\mu}^{(n)}+i\left(R / n^{2}\right) \partial_{\mu}\left(n^{m} A_{m}^{(n)}\right)$ are massive gauge fields on the brane (with mass $|n / R|)$ which are invariant (covariant in the non-abelian case) under the gauge transformation in $D$ dimensions. Since the induced gauge fields only appear in the minimal interactions to brane fields, the second term can be absorbed by a redefinition of those fields (a gauge transformation). In the following, the Kaluza-Klein gauge fields $A_{\mu}^{(n)}$ should be understood as this massive gauge field $\widetilde{A}_{\mu}^{(n)}$. It should be noted that in the above equation, the zero mode part contains the zero modes of extra components $A_{m}$ which remains massless. Since such massless 'scalar' fields in the adjoint representation have not been experimentally observed, they must be removed from the low-energy effective theories. Of course, it is not necessary that gauge fields are living in the bulk unlike the graviton. The effects of the NG field, which we will discuss below, actually exist even when the gauge fields are confined on the brane to start with. However, if one wish to consider bulk gauge fields, one should incorporate some mechanism, such as non-trivial compactifications, in order to remove such adjoint scalars.

\section{Effects of the NG boson $\phi$}

In this section, we investigate the effects of the Nambe-Goldstone field $\phi(x)$ on our four-dimensional world using the action obtained in the previous section. We take the dimension of our brane $d=4$ and denote the number of extra dimensions $(D-d) \equiv \delta$. 
It is also simply assumed that as in the usual case, the gravity (and sometimes the standard gauge fields, too) live in the bulk and the other matter fields are all confined in the four-dimensional brane. For simplicity, we use a dimension one parameter $f$ for the brane tension in the following:

$$
\tau \equiv f^{4} / 4 \pi^{2}
$$

Strictly speaking, when we take the bulk gravity turned on, the NG bosons $\phi^{m}$ are absorbed by the zero modes of the off-diagonal components of the bulk metric, $B_{\mu}^{m}$, and the latter become massive. Their masses, however, become of the order $\sim \sqrt{\tau} / M_{\mathrm{pl}}\left[12\right.$ ( $M_{\mathrm{pl}}$ is the Planck mass in four dimensions), and very small compared to the energy scales which we will consider. Therefore, we need not take into account this Higgs effect thanks to the equivalence theorem [13], and will treat $\phi$ (and graviton zero modes) to be massless fields.

\subsection{Suppressions of KK mode couplings}

First, we briefly review the results of Ref. [8] about the couplings (3.10) between the brane fields and the bulk ones. As we will see below, the couplings can exponentially reduce the contributions of higher KK modes and potentially make their effects invisible in our four-dimensional world.

The form of the interaction term (3.10) now implies that the effective coupling $g_{n}$ of the level $n$ KK mode to four-dimensional fields is actually suppressed exponentially:

$$
g_{n} \equiv g \cdot e^{-\frac{1}{2}\left(\frac{n}{R}\right)^{2} \frac{M^{2}}{f^{4}}} .
$$

The origin of this suppression is a recoil effect of the brane. This is easily seen if we note that the effective couplings $g_{n}$ can be written as

$$
g_{n}=g \cdot\left\langle 0\left|e^{2 \pi i \frac{n}{R} \frac{\phi(x)}{f^{2}}}\right| 0\right\rangle,
$$

by using the perturbative vacuum $|0\rangle$ of the NG bosons $\phi$. Remembering that $\phi(x)$ stand for the transverse coordinate, the operator $e^{i \frac{n}{R} \phi(x)}$ is just like the vertex operator in the string theory and gives transverse momentum $n / R$ to the brane around the point $x$. Hence, $e^{2 \pi i \frac{n}{R} \frac{\phi(x)}{f^{2}}}|0\rangle$ represents the recoiled state of $\phi$ by the absorption (emission) of the level $n$ KK mode carrying transverse momentum $n / R(-n / R)$. Thus the amplitude $\left\langle 0\left|e^{2 \pi i \frac{n}{R} \frac{\phi(x)}{f^{2}}}\right| 0\right\rangle$ can be viewed as a probability amplitude of containing the 
original state $|0\rangle$ in the recoiled state $e^{2 \pi i \frac{n}{R} \frac{\phi(x)}{f^{2}}}|0\rangle$. As is clear from this view, the suppressions become stronger for higher KK modes since larger deformation of the brane occurs, and on the other hand, in the case of stiff brane possessing large $f$, the suppression is weak.

Let us discuss a phenomenological consequence of this new suppression factor. Consider a tree-level amplitude for the two charged-particle scattering in the brane caused by the exchange of KK gauge bosons which live in the bulk. This leads to a correction to the effective four-Fermi coupling constant $G_{F}$. The summation over all the KK modes in the amplitude becomes

$$
\sum_{n} g_{n}^{2}\left\langle A_{\mu}^{(n)} A_{\nu}^{(-n)}\right\rangle \sim \sum_{n} g_{n}^{2} \frac{1}{M_{W}^{2}+n^{2} / R^{2}},
$$

where we have assumed the momentum transfer is small compared with $M_{W}$ (and then $R^{-1}$ ). If the couplings of KK modes were universal, ${ }^{\forall} g_{n}=g$, this amplitude diverges when the dimensions transverse to the brane are greater than one. This is something wrong because it is merely a tree-level amplitude and the divergence cannot be renormalized by any means. In the recent analyses on the experimental implications of such KK modes contributions, the sum has simply been cut off at the fundamental scale $M$. However, we see above that such divergences are automatically cured as it should by properly taking into account the brane fluctuations (the fluctuations of $\phi$ ). ..

For a numerical estimation, we simply consider the $\delta=1$ case, but it is straightforward to include more numbers of extra dimensions. The correction (4.4) is dominated by the first mode contributions (with $n= \pm 1$ ) since the higher modes are further suppressed by the presence of exponential factors, so that the KK mode correction $\Delta G_{F}$ to the four-Fermi coupling constant can be estimated as

$$
\frac{\Delta G_{F}}{G_{F}} \sim 2 \frac{g_{n=1}^{2}}{g^{2}} \frac{M_{W}^{2}}{M_{W}^{2}+1 / R^{2}} .
$$

Since the standard model prediction precisely agrees with experiments, the KK mode correction (4.5) must be small, say $\lesssim O\left(10^{-2}\right)$ [15]. For definiteness, we consider $R^{-1}$ in the range $M_{W}<R^{-1}(<M)$, then the constraint $\Delta G_{F} / G_{F}<10^{-2}$ reads

$$
2 M_{W}^{2} R^{2} \cdot e^{-\frac{M^{2}}{R^{2} f^{4}}}<10^{-2} .
$$

This constraint gives a weak upper bound to the brane tension; $f \lesssim O(1) \mathrm{TeV}$, depending on the value of $M$. Clearly, since $\exp \left[-\left(M^{2} / R^{2} f^{4}\right)\right]<1$, no constraint

* Similar suppression factors are also discussed in different frameworks [14]. 
appears if $R^{-1}$ is larger than $10 \sqrt{2} M_{W} \sim 1.1 \mathrm{TeV}$. Even for $R^{-1}$ less than this, the constraint (4.6) can be easily satisfied for any value of $M$ provided that $f$ is chosen suitably small. Therefore, if the brane fluctuation is taken into account, the constraints on the extra dimensions $(R, M)$ so far obtained can be substantially loosened and sometimes disappear. In the case of $\delta \geq 2$, since the exponential damping factor appears for each extra dimension, it is clear that the suppression factor becomes $\left(g_{n} / g\right)^{2 \delta}$ and then, the constraint of $f$ is further weaker.

Finally, it should be noted that the exponential suppressions discussed above also work in the couplings of the KK gravitons to the matters on the brane. This fact will have important effects in later analyses.

\subsection{The fifth force}

In the previous section, we have shown that the contributions from all KK excited modes are suppressed if the brane tension $f$ takes a moderately small value. Then, we unfortunately could not find signatures of the presence of large extra dimensions unless there is a lower bound for $f$. In section 3, we have seen two types of interaction terms for the NG field $\phi(x)$ involving the coupling $f$. One is the exponential couplings between the brane fields and the bulk ones whose effect was discussed before. It gave only upper bounds of $f$. Expanding the exponential interaction, we also have the $n$ NG boson coupling whose strength in general takes the form $\sim\left(1 / R f^{2}\right)^{n} \exp \left[-M^{2} / R^{2} f^{4}\right]$. This factor takes a maximum value at $f \sim \sqrt{M / R}$, which is independent of $f$, and so cannot be used to give a lower bound for $f$ either. On the other hand, another type of couplings in the lagrangian (3.4), coming from the expansion of the vierbein, has strength proportional to $f^{-2}$. As the brane tension becomes smaller, its effects become larger and may easily be detected. In the following two sections, we will calculate various phenomena which involve the latter coupling, and show that relatively severe lower bounds can be obtained.

First, we discuss a constraint from the so-called "fifth force". So far, various types of new long range forces mediated by hypothesized very light particles have been suggested in many theoretical frameworks [16]. We here consider the strength of a long range force which arises as a consequence of exchanges of the NG scalar $\phi$, which could be potentially observable. In the macroscopic range, the dominant force working between neutral systems is gravity. The Newton's universal law of gravitation (the inverse-square distance dependence) has been experimentally tested up to $1 \mathrm{~cm}$ range, 
and new forces which are comparable to gravity have been excluded to this range [17]. This fact restricts the potential forms of new forces and imposes the bounds for new couplings which determine the potentials. In the present model, this just results in a lower bound for $f$. As seen from the effective lagrangian on the brane, the NG mediated force between two distinguishable standard fermions with masses $m$ and $m^{\prime}$ is calculated from the one-loop diagram in the leading order of $f^{-1}$ (Fig. 1). Note

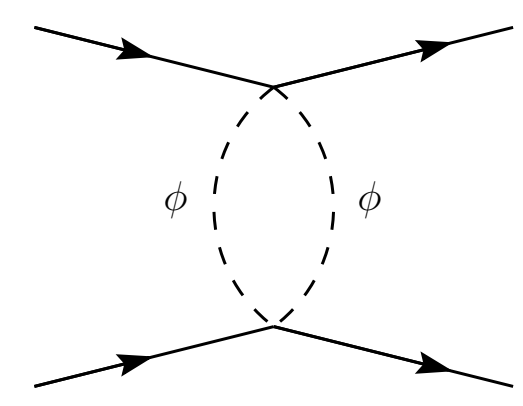

Figure 1: The lowest-order contribution to the NG boson mediated force.

that since a single $\phi$ does not have tree-level couplings to the standard fermions, this two-particle exchange is the leading contribution. This is because in the action on the brane, there is an $S O(\delta)$ internal symmetry under which $\phi$ transform as a vector. So, unlike the case of Yukawa force mediated by massless scalars, the above new force at a distance $r$ is expected to behave as $1 / r^{n}(n>2)$. We calculate the above amplitude in the non-relativistic limit and extract the two-body potential $V(r)$ from it. When we now consider a loop diagram and a higher-order correction to gravity, a convenient way to compute the two-body potential is the dispersion theoretical method [18]. This method gives a following relation between the potential $V(r)$ and invariant scattering amplitude $\mathcal{M}$ obtained from Feynman diagrams,

$$
V(r)=\frac{i}{8 \pi^{2} r} \int_{0}^{\infty} d t \mathcal{M}_{t} \exp (-\sqrt{t} r)
$$

where $t$ denotes the momentum transfer (that is now used beyond the physical region) and $\mathcal{M}_{t}$ is the discontinuity of amplitude across the branch cut on the real $t$ axis; $\left.\mathcal{M}_{t} \equiv 2 i \operatorname{Im} \mathcal{M}(t+i \epsilon)\right|_{\epsilon \rightarrow+0}$. Therefore, in order to obtain the expressions for long range potentials we have only to calculate the $t$-channel discontinuity and perform a Laplace transformation. The discontinuity of amplitudes can be evaluated by a simple prescription resulting in the replacement of each propagator by its discontinuity (delta 
function) multiplied by a theta function to insure a positive energy [18;

$$
\frac{1}{m^{2}-k^{2}} \longrightarrow 2 \pi i \delta\left(m^{2}-k^{2}\right) \theta\left(k^{0}\right)
$$

For the above diagram, we obtain for $\mathcal{M}_{t}$ in the non-relativistic limit neglecting the higher-order contributions of three-momenta,

$$
\mathcal{M}_{t}=\frac{\pi^{3} i \delta m m^{\prime}}{80 f^{8}} t^{2}
$$

where $\delta$ is the number of extra dimensions. We here have used a formula

$$
\int d^{4} k \delta\left(k^{2}\right) \delta\left((k-q)^{2}\right) k^{\mu} k^{\nu} k^{\rho} k^{\sigma}=\frac{\pi}{10}\left(q^{\mu} q^{\nu} q^{\rho} q^{\sigma}-\frac{1}{8} g^{(\mu \nu} q^{\rho} q^{\sigma)} q^{2}+\frac{1}{48} g^{(\mu \nu} g^{\rho \sigma)} q^{4}\right)
$$

where two totally symmetric sums are defined as

$$
\begin{aligned}
g^{(\mu \nu} q^{\rho} q^{\sigma)} & =g^{\mu \nu} q^{\rho} q^{\sigma}+g^{\mu \rho} q^{\nu} q^{\sigma}+g^{\mu \sigma} q^{\nu} q^{\rho}+g^{\nu \rho} q^{\mu} q^{\sigma}+g^{\nu \sigma} q^{\mu} q^{\rho}+g^{\rho \sigma} q^{\mu} q^{\nu} \\
g^{(\mu \nu} g^{\rho \sigma)} & =g^{\mu \nu} g^{\rho \sigma}+g^{\mu \rho} g^{\nu \sigma}+g^{\mu \sigma} g^{\nu \rho}
\end{aligned}
$$

As a result, we see that this force has a potential

$$
V(r)=-\frac{3 \pi \delta}{8 f^{8}} \frac{m m^{\prime}}{r^{7}}
$$

implying an attractive long range force. In Appendix B, we show another derivation of this result by using more conventional method.

Before performing numerical comparisons with the experiments, we here give several comments on other possible contributions besides the above potential. First, there is a contribution from the massive KK gravitons. The deviations from the Newton's law by the KK graviton mediated force becomes Yukawa-type potentials [19 and can be negligible in the macroscopic region. On the other hand, when the number of extra dimensions is two this force can be as strong as the four-dimensional gravity at submillimeter range for the fundamental scale $M \simeq 1 \mathrm{TeV}$. (In that case, moreover, the KK graviton mediated amplitude has a logarithmic dependence on the brane tension $f$ and cannot be suppressed enough for any small values of $f$.) However, several astrophysical constraints have already excluded the region for $M$, at least, up to $O(10)$ $\mathrm{TeV}$ [20] and in addition, the KK graviton mediated force has a strong dependence of $M^{-4}$. So, we can neglect this KK graviton contributions even at the level of the proposed gravitational experiments. In the case of $\delta \geq 3$, the KK graviton force is much weaker than the gravitation due to the very short Compton wavelengths of 
the KK gravitons. There are also finite temperature corrections which, in the long distance range, could become comparable to the zero-temperature contribution calculated above. So the potential $V(r)$ may receive the correction of the factor of order $O(1)$. However, since the potential has a large power dependence on the brane tension $f$, such an $O(1)$ correction is little relevant in evaluating the constraints for $f$ numerically. We therefore neglect the finite temperature corrections.

There are some types of experiments which can test deviations from the inversesquare law of the Newtonian gravity. The torsion balance experiments give the most stringent limits on the deviations up to sub-centimeter range and have excluded the presence of new forces whose strengths are comparable to or stronger than the gravity [22]. Furthermore, the electromagnetic Casimir force have recently been measured very precisely and also can impose the constraints on new forces in the range below $10^{-4} \mathrm{~m}$ [23]. By comparing our hypothetical force (4.13) with the results of those experiments, we obtain a constraint for $f$

$$
f>O(0.1) \mathrm{GeV} \text {. }
$$

Because of the steep slope of $V(r)$ proportional to $r^{-7}$, this lower bound mainly comes from the Casimir force experiments. The proposed classical gravity experiments will further improve this bound by a factor of $O(1)$.

The bound (4.14) is a very weak one. But, it certainly shows that there exists a lower bound for the brane tension.

\subsection{Energy loss in stars}

The fifth force constraint in the previous section is too weak to give an enough bound for the brane tension $f$. However, there are other processes which actually can lead severer bounds for $f$. Since $\phi$ does not have any gauge charges on the brane, its properties can only be constrained by the missing energy arguments in the collider experiments and in astrophysics. There, one usually assumes that the energy loss occurs via the standard mechanism in form of photons and/or neutrinos. For example, if there are novel low-mass particles having weak interactions with matters, they can freely escape from the interior of stars and carry away their energy. Then it will change the course of the stellar evolution that would be expected otherwise. With this observation in various astronomical observables such as the Sun, horizontal branch stars, white dwarfs, supernovas, we can derive various types of bounds on the 
coupling strengths in the models under consideration [24]. In this section, we discuss constraints on the brane tension $f$ required from the cooling process of the neutron star in supernova explosions. That is, if there were novel particles which freely stream out of the nascent neutron star, it may rather affect the observed data of neutrino burst. This will lead to the most stringent bounds from astrophysics. The other collider signatures of energy loss carried away by the NG field $\phi$ are also important and will be discussed elsewhere.

We can obtain a rough estimation for this supernova bound by comparing naively with the axion case. Though this estimation is very rough, we can see that the astrophysical requirements actually impose severer bounds for the brane tension. The axion coupling to the ordinary matter is proportional to $1 / f_{a}$, where $f_{a}$ is the axion decay constant. The cross section of axion emission from the interior core is proportional to $1 / f_{a}^{2}$. The astrophysical constraints from the supernova observation say that $f_{a}$ should be greater than $10^{10} \mathrm{GeV}$ [24]. On the other hand, a typical coupling of the NG boson $\phi$ to matters are given by $\sim T_{S N}^{3} / f^{4}$, where $T_{S N}$ is the supernova temperature (available energy in the supernova medium). If we naively convert the axion bound to the present case, then we obtain

$$
\frac{T_{S N}^{3}}{f^{4}}<\frac{1}{10^{10} \mathrm{GeV}} \longrightarrow f \gtrsim 10^{1.5} \mathrm{GeV}
$$

for the supernova temperature $T_{S N} \sim O(10) \mathrm{MeV}$. This shows that, as anticipated, the star energy loss argument certainly gives a severer bound for the brane tension than that from the fifth force constraint.

We now perform a more detailed analysis of the energy emission rate from the supernova core. There are several channels which result in energy losses in the supernova medium. We expect that the most important contribution to this is the nucleon-nucleon bremsstrahlung process like in the axion case [25] because the supernova temperature is near the pion mass and large suppressions with the pion mass do not occur. However, this process is rather involved and there are many types of uncertainties and unknown factors, for example, with reference to the effective pion description in the supernova medium. Instead, in this paper we consider a more "clean" event, i.e., the electron-positron pair annihilation process. This contribution is surely subdominant but can be as large as that of the nucleon bremsstrahlung when we adopt a higher value of temperature in the supernova. However, we should bear in mind that more stringent bounds may be obtained by accurately taking into account the dominant process mentioned above. 
Since the energy scale considered is well below the fundamental scale $M$ and the tension $f$, there are two diagrams contributing to the energy losses from the electronpositron annihilation in the leading order in the couplings $G_{N}$ and $g_{i}\left(G_{N}\right.$ is the Newton constant and $g_{i}$ are the standard gauge couplings). They are annihilations to (a) the KK gravitons $g_{\mu \nu}^{(n)}$ and (b) the NG bosons $\phi$ (Fig. 2). We estimate the initial-

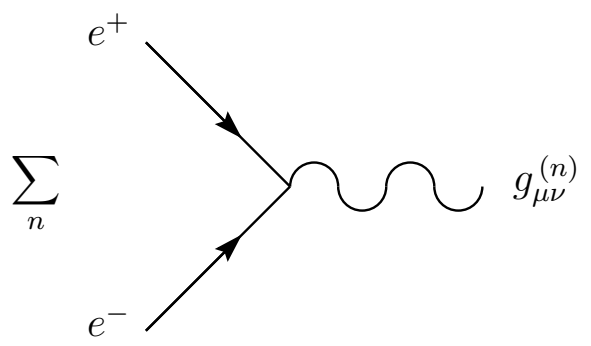

(a)

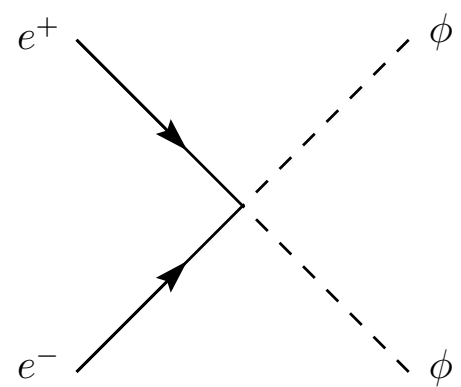

(b)

Figure 2: The energy loss processes in the supernova medium by the electron-positron annihilation: (a) KK graviton production and (b) NG boson pair production.

spin averaged cross section for each case (a) and (b). The explicit calculation is given in Appendix C. For the real KK gravitons production process, the cross section is obtained by only attaching an exponential suppression factor discussed before to the usual result:'"

$$
\begin{aligned}
\sigma_{a} & =\frac{\pi^{1-\frac{\delta}{2}}}{2^{\delta+3} \Gamma\left(\frac{\delta}{2}\right)} \frac{1}{M^{\delta+2}} s^{\frac{\delta}{2}} e^{-s \frac{M^{2}}{f^{4}}}, \\
\sigma_{b} & =\frac{\delta \pi^{3}}{1920} \frac{1}{f^{8}} s^{3}
\end{aligned}
$$

where $s$ is the center of mass energy in the electron-positron system. We have used a relation $G_{N}^{-1}=8 \pi V_{\delta} M^{\delta+2}$ where $V_{\delta}$ is the volume of the $\delta$-torus $\left(=(2 \pi R)^{\delta}\right)$. We have also neglected the electron mass for $m_{e} \ll T_{S N}$ in the core. It should be noted that the $f$-dependences of the above two cross sections are very different from each other. Typical behaviors of the cross sections are shown in Fig. 3. It can be seen from this figure that the total cross section (the solid line in Fig. 3) becomes large

\footnotetext{
${ }^{\dagger}$ If we sum up the inclusive process $e^{+}+e^{-} \rightarrow g_{\mu \nu}^{(n)}+($ any numbers of $\phi)$ in place of the exclusive process (a), the cross section $\sigma_{a}$ may be enhanced by a factor of $\exp \left(a s^{2} / f^{4}\right)$ with an $O(1)$ coefficient $a$, which is, however, not large enough to cancel the suppression factor $\exp \left(-s M^{2} / f^{4}\right)$.
} 


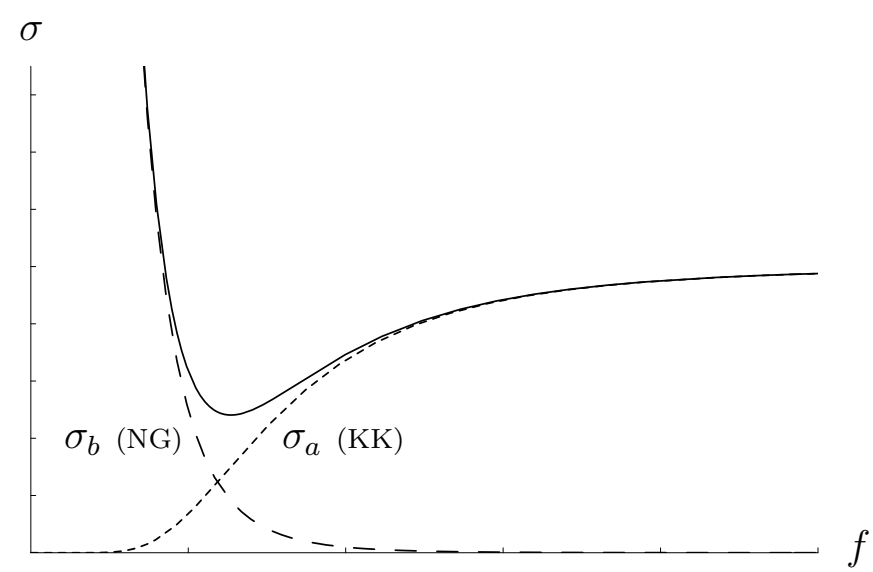

Figure 3: The typical behaviors of the cross sections against the brane tension $f$. The dotted and dashed lines denote $\sigma_{a}$ and $\sigma_{b}$, respectively. The solid line is the total cross section.

for both small and large values of the brane tension $f$. The cross section for a small value of $f$ is governed by the NG boson production process while for a large value, the KK gravitons production rate dominates the total cross section. In either way, it turns out that we cannot have arbitrarily desired values of brane tension and it will be severely restricted by phenomenological requirements.

If the present novel particles interact strongly with ordinary matters, they are scattered or absorbed again in the medium after productions in the core. Then they cannot freely escape to the outside and will be radiated from a sphere. The flux from this black-body surface emission for the neutron star becomes smaller as novel particles interact more strongly (the radius of sphere becomes larger). In that case, the region beyond some strong coupling cannot be excluded from the cooling argument like in the axion case [26]. For the KK gravitons, the energy reabsorption is, however, highly suppressed. This can be intuitively understood from the fact that the brane occupies a very tiny (almost zero) region in the whole bulk. Once the KK gravitons escape to the bulk spacetime from the brane, they will never return, at least, over the age of the universe [12]. After all, the KK gravitons can be considered to freely stream out of the core. On the other hand, since $\phi(x)$ is a field on our four-dimensional brane and does not escape to the extra dimensions, its effect may become less important than neutrinos for the strong coupling (small tension) region. For this, we estimate 
the mean free path $L$ for $\phi$ in the core, which is roughly given by

$$
L \sim \frac{f^{8}}{T_{\mathrm{SN}}^{6} n_{e}},
$$

where $n_{e}$ is the number density of electron in the core, $n_{e} \sim 10^{-3} \mathrm{GeV}^{3}$. We can see that $L$ exceeds the radius of the neutron star $\sim 10 \mathrm{~km}$ for $f \gtrsim O(10) \mathrm{GeV}$ (see, $(4.15)$ or $(4.30)$ ). Then, $\phi$ is emitted from the entire volume of the star and we can surely discuss the lower bounds of brane tension $f$. Note that if the couplings were strong and the mean free path $L$ were smaller than the star radius, other astrophysical constraints and/or too many experimental signals in the neutrino detectors [27] would reject such strong coupling regions.

From the calculated cross sections, we extract the production rates in order to compare them with the observations and to have definite conclusions about the allowed range of the parameters. However, in the hot dense medium as in the supernova, there are some uncertainties such as many-body effects, which have not been fully understood. We expect that those collective effects in the medium amount to change a factor of $O(1)$ in the production rate. This is actually the case for the axion emission from the supernova [24]. Moreover, as we will see below, the production rates have large power-dependence on parameters for which we now would like to have limits. So, even if there is an ambiguity even of factor of 10 , the final results for these parameters are not so affected.

Now, let us estimate the bounds numerically. The theory of type II supernova explosions says that in the explosion, almost all of the gravitational binding energy of a nascent neutron star is released in form of neutrinos within a few second. The expected neutrino flux and the duration of signal calculated from this picture is in good agreement with the observations for the supernova 1987A in the Kamiokande II [28] and IMB [29] detectors. This agreement implies a conservative requirement that the energy loss rate from other than neutrinos should not exceed that from neutrinos in the standard picture 24]. Otherwise the measured duration of the neutrino signal would be shorter than the observed ones. Numerically, this constraint reads

$$
Q \lesssim 10^{36} \mathrm{GeV} / \mathrm{cm}^{3} \text { sec. }
$$

Here, $Q$ is the energy loss rate per unit time and unit volume (the volume emissivity) defined as follows;

$$
Q_{x} \equiv \int \frac{d^{3} k_{1}}{(2 \pi)^{3} 2 E_{1}} \int \frac{d^{3} k_{2}}{(2 \pi)^{3} 2 E_{2}} 2 f_{1} \cdot 2 f_{2} \cdot\left(E_{1}+E_{2}\right) \cdot 2 s \sigma_{x},
$$


where $x$ is the process label, $a$ or $b$, and $f_{i}$ is the Fermi-Dirac distribution function; $f_{i}=1 /\left(\exp \left(E_{i} / T-\mu / T\right)+1\right)$ (a factor of 2 denotes the spin degrees of freedom). For $\mu$ in the distribution functions, we use the chemical potential for electron (positron) in the supernova core. Since the electron is considered as a highly degenerate relativistic particle in the core, $\mu$ is given by $\simeq\left(3 \pi^{2} n_{e}\right)^{1 / 3}$ where $n_{e}$ is the number density of electron, $n_{e} \sim 1.4 \times 10^{-3} \mathrm{GeV}^{3}$. We calculate $Q$ for each process (a) and (b), and the results become

$$
\begin{aligned}
Q_{a} & =\frac{1}{4 \pi^{\frac{\delta}{2}+3} \Gamma\left(\frac{\delta}{2}\right)} \frac{T_{S N}^{\delta+7}}{M^{\delta+2}} F_{\delta}\left(T_{S N}\right), \\
Q_{b} & =\frac{\delta}{75 \pi} \frac{T_{S N}^{13}}{f^{8}} I\left(T_{S N}\right) .
\end{aligned}
$$

The dimensionless functions $F_{\delta}(T)$ and $I(T)$ are given by

$$
\begin{aligned}
F_{\delta}(T) & \equiv \int_{0}^{\infty} \int_{0}^{\infty} d x d y \frac{(x+y)(x y)^{\delta / 2+2}}{\left(e^{x-(\mu / T)}+1\right)\left(e^{y+(\mu / T)}+1\right)} \Gamma_{\delta}\left(4 x y T^{2} M^{2} / f^{4}\right), \\
I(T) & \equiv \int_{0}^{\infty} \int_{0}^{\infty} d x d y \frac{(x+y)(x y)^{5}}{\left(e^{x-(\mu / T)}+1\right)\left(e^{y+(\mu / T)}+1\right)}
\end{aligned}
$$

where $\Gamma_{\delta}(z)$ is defined as

$$
\Gamma_{\delta}(z) \equiv \int_{0}^{1} d t t^{\delta / 2+1} e^{-z t} .
$$

Clearly, the relation $\Gamma_{\delta+2 n}(z)=(-d / d z)^{n} \Gamma_{\delta}(z)$ holds and we have

$$
\Gamma_{\delta}(z)=\left(-\frac{d}{d z}\right)^{n+1} \begin{cases}\frac{1-e^{-z}}{z} & \text { for } \delta=2 n \\ \sqrt{\frac{\pi}{z}} \operatorname{Erf}(\sqrt{z}) & \text { for } \delta=2 n-1\end{cases}
$$

where $\operatorname{Erf}(z)$ is the error function,

$$
\operatorname{Erf}(z) \equiv \frac{2}{\sqrt{\pi}} \int_{0}^{z} e^{-t^{2}} d t .
$$

From the above results, we can obtain two types of bound concerning with extra dimensions in the present model. This is mainly because the two volume emission rates have very different parameter dependences as mentioned before (see, Fig. 3). First, we discuss the lower bounds for the brane tension $f$. As can be seen from Fig. 3, for a small value of $f$, the dominant contribution to the energy loss rate is 
that from the NG bosons production. We apply the supernova constraint (4.19) for the volume emissivity to $Q_{b}$ and then obtain lower bounds (in the case of $\delta=1$ );

$$
\begin{array}{ccc}
f>8 \mathrm{GeV} & \left(T_{S N}=20 \mathrm{MeV}\right), \\
f>23 \mathrm{GeV} & \left(T_{S N}=30 \mathrm{MeV}\right), \\
& \vdots & \\
f>122 \mathrm{GeV} & \left(T_{S N}=70 \mathrm{MeV}\right) .
\end{array}
$$

Here we have adopted a rather wide range of the supernova temperature and displayed corresponding bounds for each case. Note that if there are $\delta$ extra dimensions, each value of the lower bound is multiplied by $\delta^{1 / 8}$. It is interesting that the above bounds have no dependence on the value of the fundamental scale $M$ since the cross section for NG bosons production is independent of it. The above limits are indeed $10^{2-3}$ times stronger than that from the fifth force constraint. In other words, with these bounds the long range force associated with $\phi$ is outside the reach of the proposed gravity experiments, and it also does not modify the effects of gravity in stars. In this way, the brane tension can be severely restricted from astrophysical arguments and the KK mode couplings cannot be arbitrarily suppressed. This is a welcome result for we could see signatures of the existence of extra dimensions in the near future experiments.

More interestingly, we can also get the lower bounds for the fundamental scale $M$. As depicted in Fig. 3, there is an absolute minimum of the total cross section against the brane tension $f$. That is, the star energy is necessarily carried away to a certain extent in form of the KK gravitons and NG bosons. At the minimum, the value of $f$ and then the minimum value of the energy loss rate are determined by a given value of $M$. Therefore, as a conservative bound for $M$, we require that the total energy loss rate at the minimum must not violate the supernova condition (4.19). It is clear that the value of $f$ minimizing the emission rate satisfies the above obtained lower bounds, and moreover the resultant bounds for $M$ become weaker than that naively estimated in the $\mathrm{TeV}$-scale quantum gravity scenario [20], i.e., without the coupling suppression by the brane fluctuations taken into account. The cross section for the KK graviton emissions (and hence $Q_{a}$ ) rather depends on the number of extra dimensions. It can be easily seen that the $\delta=2$ case gives the maximum cross section and hence we obtain the most restrictive bound. For $\delta>2$, the cross sections are considerably reduced with increasing power of $M^{-1}$, and we only obtain very weak lower bounds 
for $M$. The fundamental scale dependences of the energy loss rate at the minimum are shown in Fig. 4 for the $\delta=2$ case. Remarkably, the fundamental scale $M$ is strongly restricted even when our four-dimensional brane is relatively soft; e.g., $M>O(1)$ $\mathrm{TeV}$ for $T_{S N}=30 \mathrm{MeV}$. Notice that we may have more severe bounds for parameters

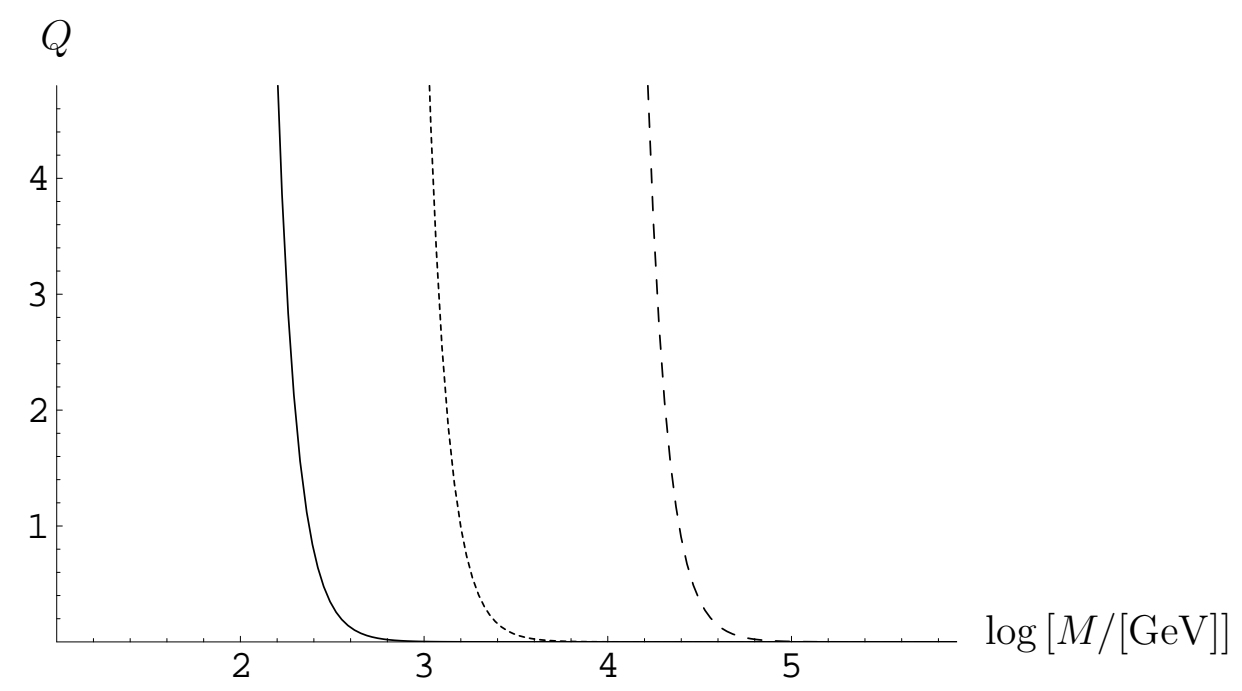

Figure 4: The volume emissivity $Q$ in the supernova core for $\delta=2$ case. The solid, dotted and dashed line correspond to $T_{S N}=20,30,70 \mathrm{MeV}$, respectively. ( $Q$ is denoted in units of $10^{36} \mathrm{GeV} / \mathrm{cm}^{3}$ sec.)

if we consider the dominant process of nucleon bremsstrahlung in the supernova core as noted before, and moreover other experimentally observable processes may give considerable impacts on the model parameters. Anyway, we cannot have arbitrarily small values for the brane tension and therefore do not miss the possibility of finding signatures of the extra dimensions.

\section{Summary}

In this paper, we have investigated the possibility where our four-dimensional world is embedded as a brane in higher dimensional spacetime. In such a situation, the translational symmetry transverse to the brane is spontaneously broken by the presence of brane itself. As a consequence, the massless NG fields appear which denote the position of brane in the higher dimensional bulk. For such a setup, the effective action 
for the four-dimensional fields is described by using the induced metric and vierbein on the brane. These induced quantities can be given in terms of the bulk vielbein and the NG variables. We have found the explicit expression for the induced vielbein and written down the effective action for fermion fields confined on our brane. Given the interactions, we can discuss phenomenological implications of the brane effective action, especially, several interesting effects of the NG fields.

First, we discussed the KK mode couplings to the brane. We found in [8 that higher KK mode contributions are exponentially suppressed with a suitably small tension of our world, i.e., for a relatively soft brane. However, some other phenomenological observations can impose limits on this softness. We have shown in practice that severe lower bounds for the brane tension can be obtained by considering, for example, the null observations of the fifth force, the missing energy process in the supernova explosions such as SN1987A, and so on. The existence of these lower bounds implies that the KK mode contributions cannot be arbitrarily suppressed and could be observable in the near future experiments as has been discussed so far in many articles. We have also been able to calculate a lower bound for the fundamental scale of the effective theory of our brane. More promising and severe bounds for the physics of extra dimensions (the fundamental scale, the compactification radius, etc.) would be obtained by studying their signatures in the collider experiments and other astrophysical and cosmological requirements. Hence, it will be an interesting and challenging subject to find evidences for the existence of extra spacetime dimensions in form of the Kaluza-Klein modes and/or the NG bosons appearing on our four-dimensional world.

\section{Acknowledgements}

We would like to thank M. Bando, K. Hashimoto, T. Hirayama, T. Noguchi, N. Sugiura for valuable discussions and comments. T. K. and K. Y. are supported in part by the Grants-in-Aid for Scientific Research No. 10640261 and the Grant-in-Aid No. 9161, respectively, from the Ministry of Education, Science, Sports and Culture, Japan. 


\section{A Induced vielbein}

In this appendix, we derive an explicit form of the induced vielbein on the $d$-dimensional brane. It is written in terms of the $D$-dimension bulk vielbein and the $(D-d) \mathrm{NG}$ variables denoting the position of brane in the bulk.

The $d$-dimensional brane breaks the bulk Lorentz group $G=S O(1, D-1)$ down to $H=S O(1, d-1) \times S O(D-d)$. Consider the coset-space variable $\xi(\theta) \in G / H$,

$$
\xi(\theta(x))=\exp \left(i \theta_{a}^{\alpha}(x) \eta_{\alpha \beta} J^{(\beta a)}\right)
$$

with broken generators $J^{(\alpha a)}$. The fields $\theta_{a}^{\alpha}(x)$ is the NG boson associated with the spontaneous breaking of $G$ down to $H$. Act the bulk Lorentz transformation $g \in G$ to the element $\xi(\theta(x))$ from the left, then the resultant $g \xi(\theta(x))$ is still an element of $G$ and can be decomposed uniquely into the form:

$$
g \xi(\theta(x))=\xi\left(\theta^{\prime}(x)\right) h(g, \theta(x)), \quad{ }^{\exists} h(g, \theta(x)) \in H .
$$

Thus, the bulk Lorentz transformation $g \in G$ for the NG variable $\theta(x)$ is defined by

$$
\xi(\theta(x)) \stackrel{g}{\longrightarrow} \xi\left(\theta^{\prime}(x)\right)=g \xi(\theta(x)) h^{-1}(g, \theta(x)) .
$$

An important property of $\xi(\theta(x))$ is that the $G$ transformation $g$ is converted through $\xi(\theta(x))$ into the corresponding $H$ transformation $h(g, \theta(x))$.

Up to here everything is the standard story of the nonlinear realization theory [30]. We wish to define the vielbein $e^{\alpha}{ }_{\mu}$ induced on the brane from the bulk vielbein $E^{A}{ }_{M}$. The necessary conditions for the desired induced vielbein $e^{\alpha}{ }_{\mu}$ to satisfy are that (i) $e^{\alpha}{ }_{\mu}$ for each $\mu$ transforms as a $d$-dimensional vector under $S O(1, d-1)$. (ii) the metric given by $e^{\alpha}{ }_{\mu}$ should coincides with the induced metric;

$$
g_{\mu \nu}=\eta_{\alpha \beta} e_{\mu}^{\alpha} e_{\nu}^{\beta}=G_{M N} \partial_{\mu} Y^{M} \partial_{\nu} Y^{N}=\eta_{A B} E_{M}^{A} E_{N}^{B} \partial_{\mu} Y^{M} \partial_{\nu} Y^{N}
$$

The conversion of the curved index $M$ in the bulk into $\mu$ on the brane can easily be done using a bulk vector tangent to the brane:

$$
\mathcal{E}_{\mu}^{A}=E_{M}^{A} \partial_{\mu} Y^{M}
$$

This defines $d$ tangent $D$-dimensional vectors which transform linearly under $g \in$ $S O(1, D-1)$. What we want now is $e^{\alpha}{ }_{\mu}$ which gives $d$ tangent $d$-dimensional vectors of $S O(1, d-1)$. We already know that $\xi(\theta(x))$ converts the $g \in G$ rotation into 
the $h \in H$ rotation; $g \xi(\theta(x))=\xi\left(\theta^{\prime}(x)\right) h(g, \theta(x))$. Therefore the following quantity defined by

$$
e_{\mu}^{A} \equiv \xi^{-1}(\theta(x))_{B}^{A} \mathcal{E}_{\mu}^{B}
$$

for each fixed $\mu$, receives only a (non-linear) $H$ rotation under $G$ transformation,

$$
e_{\mu}^{\prime}=\xi^{-1}\left(\theta^{\prime}(x)\right) \mathcal{E}_{\mu}^{\prime}=h(g, \theta(x)) \xi^{-1}(\theta(x)) g^{-1} \cdot g \mathcal{E}_{\mu}=h(g, \theta(x)) e_{\mu} .
$$

So, $e^{A}{ }_{\mu}$ splits into a $d$-dimensional vector $e^{\alpha}{ }_{\mu}$ of $S O(1, d-1)$ and a $(D-d)$-dimensional vector $e^{a}{ }_{\mu}$ of $S O(D-d)$. Thus, the former $d d$-dimensional vectors $e_{\mu}^{\alpha}$ satisfy the property (i) which is required for our desired vielbein on the brane. Note here that since $\xi^{-1}(\theta(x))$ itself is an $S O(1, D-1)$ rotation,

$$
\begin{aligned}
\eta_{A B} e_{\mu}^{A} e_{\nu}^{B} & =\eta_{\alpha \beta} e_{\mu}^{\alpha} e_{\nu}^{\beta}+\eta_{a b} e_{\mu}^{a} e^{b}{ }_{\nu} \\
& =\eta_{A B} \mathcal{E}_{\mu}^{A} \mathcal{E}_{\nu}^{B}=\eta_{A B} E^{A}{ }_{M} E^{B}{ }_{N} \partial_{\mu} Y^{M} \partial_{\nu} Y^{N}
\end{aligned}
$$

Therefore, in order to satisfy the property (ii), the remaining $(D-d)$-dimensional vector components $e^{a}{ }_{\mu}$ must vanish (see, Eq. (A.4)):

$$
e_{\mu}^{a}=\xi^{-1}(\theta(x))^{a}{ }_{B} \mathcal{E}_{\mu}^{B}=0
$$

This condition is the same as given by Sundrum [9]. It should be noted that this requirement is invariant under $G=S O(1, D-1)$ transformation since $e_{\mu}^{a}$ receives only $S O(D-d)$ rotation under $G$. The condition (A.9) gives a constraint on the NG variable $\theta$. Actually, the number of equations in (A.9) is $d(D-d)$ which is just the same as the number of $\theta$ fields. Thus the NG variables $\theta$ are completely determined in terms of $\mathcal{E}_{\mu}^{A}=E^{A}{ }_{M} \partial_{\mu} Y^{M} ; \theta=\theta(\mathcal{E})$. Although $\theta$ has now become dependent variables $\theta(\mathcal{E})$, the transformation property under $g \in G$ still remains the same as above. That is, $\theta^{\prime}=\theta(g \mathcal{E})$ holds because the constraint (A.9) is $G$-invariant. With this $\theta(\mathcal{E})$, the desired induced vielbein $e_{\mu}^{\alpha}$ is given by

$$
e^{\alpha}{ }_{\mu}=\xi^{-1}(\theta(\mathcal{E}))_{A}^{\alpha} \mathcal{E}_{\mu}^{A}
$$

It is a difficult problem to give an explicit form for the solution $\theta(\mathcal{E})$. However, what we actually need is not $\theta(\mathcal{E})$ but the induced vielbein $e^{\alpha}{ }_{\mu}$. We can find the explicit form for it as follows. 
Using the explicit representation of $D$-dimensional Lorentz generators $\left(J^{(A B)}\right)_{D}^{C}=$ $i \eta^{C E}\left(\delta_{E}^{A} \delta_{D}^{B}-\delta_{D}^{A} \delta_{E}^{B}\right)$, the coset-space variable can be written as

$$
\begin{gathered}
\xi^{-1}(\theta)=\exp \left(\begin{array}{cc}
0 & \theta \\
-\tilde{\theta} & 0
\end{array}\right)={ }_{D-d}^{d}\left(\begin{array}{cc}
C(\theta \tilde{\theta}) & S(\theta \tilde{\theta}) \theta \\
-\tilde{\theta} S(\theta \tilde{\theta}) & C(\tilde{\theta} \theta)
\end{array}\right), \\
\theta=\left(\theta_{a}^{\alpha}\right), \quad \tilde{\theta}_{\beta}^{a} \equiv \eta^{a b}\left(\theta^{\mathrm{T}}\right)_{b}{ }^{\alpha} \eta_{\alpha \beta},
\end{gathered}
$$

where we have introduced the functions $C(x)$ and $S(x)$, similar to usual cosine and sine:

$$
\begin{aligned}
C(x) & \equiv \sum_{n=0}^{\infty} \frac{(-1)^{n}}{(2 n) !} x^{n}=\cos \sqrt{x} \\
S(x) & \equiv \sum_{n=0}^{\infty} \frac{(-1)^{n}}{(2 n+1) !} x^{n}=\frac{\sin \sqrt{x}}{\sqrt{x}}
\end{aligned}
$$

When we write the $D$-dimensional vectors $\mathcal{E}_{\mu}^{A}$ in the following form splitting the parallel and transverse components to the brane,

$$
\mathcal{E}_{\mu}^{A}=\left(\begin{array}{c}
\mathcal{E}_{\| \mu}^{\alpha} \\
\mathcal{E}_{\perp \mu}^{a}
\end{array}\right)
$$

the constraint (A.9) reads

$$
(\tilde{\theta} S(\theta \tilde{\theta}))_{\beta}^{a} \mathcal{E}_{\| \mu}^{\beta}=C(\tilde{\theta} \theta)^{a}{ }_{b} \mathcal{E}_{\perp \mu}^{b}
$$

and the induced vielbein $(\underline{A .10})$ becomes

$$
e_{\mu}^{\alpha}=C(\theta \tilde{\theta})_{\beta}^{\alpha} \mathcal{E}_{\| \mu}^{\beta}+(S(\theta \tilde{\theta}) \theta)_{b}^{\alpha} \mathcal{E}_{\perp \mu}^{b} .
$$

In the above, $C(\theta \tilde{\theta})_{\beta}^{\alpha}, C(\tilde{\theta} \theta)^{a}{ }_{b}$ and $\mathcal{E}_{\| \mu}^{\alpha}$ are all square matrices and invertible. Then we obtain from Eq. (A.15),

$$
(T(\tilde{\theta} \theta) \tilde{\theta})_{\beta}^{a}=\mathcal{E}_{\perp \mu}^{a} \mathcal{E}_{\|}^{-1 \mu},
$$

where $T(x)$ is a function analogous to tangent:

$$
T(x) \equiv C^{-1}(x) S(x)=\frac{\tan \sqrt{x}}{\sqrt{x}} .
$$

In deriving Eq. (A.17), we have used the "shifting identities" like

$$
\tilde{\theta} F(\theta \tilde{\theta})=F(\tilde{\theta} \theta) \tilde{\theta}, \quad \theta F(\tilde{\theta} \theta)=F(\theta \tilde{\theta}) \theta
$$


which generally hold for any function $F(x)$. The equation (A.17) determines $\theta$ in terms of $\mathcal{E}$. To obtain an expression of the induced vielbein $e^{\alpha}{ }_{\mu}$, we define the following quantity:

$$
\mathcal{M} \equiv \eta\left(\mathcal{E}_{\perp} \mathcal{E}_{\|}^{-1}\right)^{\mathrm{T}} \eta \mathcal{E}_{\perp} \mathcal{E}_{\|}^{-1}=\theta T(\tilde{\theta} \theta) T(\tilde{\theta} \theta) \tilde{\theta}=\theta \tilde{\theta} T^{2}(\theta \tilde{\theta})
$$

Using $x T^{2}(x)+1=C^{-2}(x)$, we further find

$$
C(\theta \tilde{\theta})=(1+\mathcal{M})^{-1 / 2}
$$

Thus, the induced vielbein (A.16) is finally rewritten as

$$
\begin{aligned}
e_{\mu}^{\alpha} & =[C(\theta \tilde{\theta})]_{\gamma}^{\alpha}\left(1+(T(\theta \tilde{\theta}) \theta)\left(\mathcal{E}_{\perp} \mathcal{E}_{\|}^{-1}\right)\right)_{\beta}^{\gamma} \mathcal{E}_{\| \mu}{ }^{\beta}{ }^{2} \\
& =(1+\mathcal{M})^{\frac{1}{2} \alpha}{ }_{\beta} \mathcal{E}_{\| \mu}{ }^{\beta} .
\end{aligned}
$$

This, with the definition of $\mathcal{M}$ in Eq. (A.20), gives the desired explicit expression for the induced vielbein. If we use the shifting identity, we can rewrite it into a slightly more convenient expression as

$$
\begin{aligned}
e_{\mu}^{\alpha} & =\mathcal{E}_{\| \nu}^{\alpha}(1+\mathcal{N})^{1 / 2 \nu}{ }_{\mu}, \\
\mathcal{N} & \equiv\left(\mathcal{E}_{\|}^{\mathrm{T}} \eta \mathcal{E}_{\|}\right)^{-1}\left(\mathcal{E}_{\perp}^{\mathrm{T}} \eta \mathcal{E}_{\perp}\right)
\end{aligned}
$$

Finally in this Appendix, we add the expression for the induced spin connection $\omega_{\mu}^{\alpha}{ }_{\beta}(x)$ from the bulk one $\Omega_{M}{ }_{B}{ }_{B}(X)$. From the bulk local-Lorentz transformation law for the covariant derivative

$$
\partial_{M}+i \Omega_{M}^{\prime}(X)=g(X)\left(\partial_{M}+i \Omega_{M}(X)\right) g^{-1}(X)
$$

and the fact that $\xi$ is a converter of the local-Lorentz indices from bulk to brane, it is clear that the quantity

$$
\omega_{\mu \beta}^{\alpha}(x)=-i\left[\xi^{-1}\left(\partial_{\mu}+i \Omega_{\mu}\right) \xi\right]_{\beta}^{\alpha}=\left(\xi^{-1}\right)_{A}^{\alpha} \Omega_{\mu B}^{A}(x) \xi_{\beta}^{B}-i\left(\xi^{-1} \partial_{\mu} \xi\right)_{\beta}^{\alpha}
$$

with $\Omega_{\mu}{ }_{B}^{A}(x) \equiv \Omega_{M}{ }_{B}^{A}(Y(x)) \partial_{\mu} Y^{M}(x)$, transforms as

$$
\omega_{\mu}(x) \rightarrow \omega_{\mu}^{\prime}(x)=h(x) \omega_{\mu}(x) h^{-1}(x)-i h(x) \partial_{\mu} h^{-1}(x),
$$

with $h$ determined by (A.2). This is just the local-Lorentz transformation induced on the brane, and thus Eq. (A.25) gives the desired induced spin connection on the brane. 
If the bulk connection is the usual one $\Omega_{M}(E)$ given in terms of the vielbein $E^{A}{ }_{M}$ as the solution of $\partial_{M} E^{A}{ }_{N}-\Omega_{M}{ }_{B} E^{B}{ }_{N}-(M \leftrightarrow N)=0$, then, the induced connection (A.25) is also seen to equal the usual one $\omega_{\mu}(e)$ given in terms of the induced vierbein $e^{\alpha}{ }_{\mu}$. For completeness, we cite the explicit expression of $\xi^{-1}$ in terms of $\mathcal{E}$ :

$$
\xi^{-1}(\theta(\mathcal{E}))=\left(\begin{array}{cc}
(1+\mathcal{M})^{-1 / 2} \alpha_{\beta} & {\left[(1+\mathcal{M})^{-1 / 2} \eta\left(\mathcal{E}_{\perp} \mathcal{E}_{\|}^{-1}\right)^{\mathrm{T}} \eta\right]_{b}^{\alpha}} \\
-\left[\left(1+\mathcal{M}^{\prime}\right)^{-1 / 2} \mathcal{E}_{\perp} \mathcal{E}_{\|}^{-1}\right]_{\beta}^{a} & \left(1+\mathcal{M}^{\prime}\right)^{-1 / 2}{ }_{b}{ }_{b}
\end{array}\right)
$$

where $\mathcal{M}^{\prime} \equiv \mathcal{E}_{\perp} \mathcal{E}_{\|}^{-1} \eta\left(\mathcal{E}_{\perp} \mathcal{E}_{\|}^{-1}\right)^{\mathrm{T}} \eta$. (Note that $F\left(\mathcal{M}^{\prime}\right) \mathcal{E}_{\perp} \mathcal{E}_{\|}^{-1}=\mathcal{E}_{\perp} \mathcal{E}_{\|}^{-1} F(\mathcal{M})$ by shifting identity.) $\xi$ is given simply by changing the signs of the off-diagonal elements.

\section{B The potential of the fifth force}

Let us first evaluate the amplitude for the diagram in Fig. 1. If the external matter fields are on the mass shell, only the part $+(1 / 2 \tau)\left(\partial^{\mu} \phi^{m} \partial_{\nu} \phi^{m}\right)\left(\bar{\psi} i \gamma^{\nu} \partial_{\mu} \psi\right)$ of the interaction Lagrangian (3.4) contributes since the remaining parts vanish by the equation of motion for $\psi$. Then the amplitude is given by

$$
\begin{aligned}
& \mathcal{M}=\frac{\delta}{4 \tau^{2}}\left(\bar{u}\left(p_{3}\right) \gamma_{\mu} \frac{\left(p_{1}+p_{3}\right)_{\nu}}{2} u\left(p_{1}\right)\right)\left(\bar{u}\left(p_{4}\right) \gamma_{\rho} \frac{\left(p_{1}+p_{3}\right)_{\sigma}}{2} u\left(p_{2}\right)\right) \times I^{\mu \nu \rho \sigma}(q), \\
& I^{\mu \nu \rho \sigma}(q)=\int \frac{d^{n} k}{i(2 \pi)^{n}} \frac{k^{\mu} k^{\rho}(k+q)^{\nu}(k+q)^{\sigma}+k^{\nu} k^{\rho}(k+q)^{\mu}(k+q)^{\sigma}}{k^{2}(k+q)^{2}}
\end{aligned}
$$

where $q$ is the momentum transfer $q=p_{3}-p_{1}=p_{2}-p_{4}$ and $n=4-2 \varepsilon$. The terms in $I^{\mu \nu \rho \sigma}(q)$, in which any vector indices of $\mu, \nu, \cdots$ are carried by $q$, cannot contribute to the amplitude because of the conservation of the energy momentum tensor $(1 / 2) \bar{u}\left(p_{i}\right) \gamma_{\nu}\left(p_{j}+p_{i}\right)_{\mu} u\left(p_{j}\right)$. So, the only term we have to compute is the one proportional to $g^{(\mu \nu} g^{\rho \sigma)} \equiv g^{\mu \nu} g^{\rho \sigma}+g^{\mu \rho} g^{\nu \sigma}+g^{\mu \sigma} g^{\rho \nu}$ which comes solely from the $k^{\mu} k^{\nu} k^{\rho} k^{\sigma}$ term in $I^{\mu \nu \rho \sigma}(q)$, and we find

$$
\begin{aligned}
\left.I^{\mu \nu \rho \sigma}(q)\right|_{g^{(\mu \nu} g^{\rho \sigma)} \text { term }} & =\Gamma(\varepsilon-2) \int_{0}^{1} d x\left(\frac{-x(1-x) q^{2}}{4 \pi}\right)^{2-\varepsilon} \frac{1}{2} g^{(\mu \nu} g^{\rho \sigma)} \\
& =\frac{1}{64 \pi^{2}} g^{(\mu \nu} g^{\rho \sigma)} \frac{\left(q^{2}\right)^{2}}{30}\left(C-\ln \left(-q^{2}\right)\right),
\end{aligned}
$$

where $C$ is a divergent constant

$$
C=\frac{1}{\bar{\varepsilon}}+\frac{3}{2}+\frac{47}{30}, \quad\left(\frac{1}{\bar{\varepsilon}} \equiv \frac{1}{\varepsilon}-\gamma+\ln 4 \pi\right) .
$$

This divergent term is absorbed into a term $g^{(\mu \nu} g^{\rho \sigma)} \square\left[\bar{\psi} i \gamma_{\mu}\left(\overleftrightarrow{\partial}_{\nu} / 2\right) \psi\right] \square\left[\bar{\psi} i \gamma_{\rho}(\stackrel{\leftrightarrow}{\partial} \sigma / 2) \psi\right]$ which is to appear in the higher-dimensional terms of our effective Lagrangian. (This 
is the renormalization of the effective theory à la Weinberg.) Thus the factor $\left(C-\ln \left(-q^{2}\right)\right)$ is replaced by a finite one $-\ln \left(-q^{2} / \mu^{2}\right)$ with a suitable renormalization scale $\mu$.

In the low energy limit, the amplitude is dominated by the $\mu=\nu=\rho=\sigma=0$ components and reduces to

$$
\mathcal{M}=\frac{m m^{\prime} \delta}{4 \tau^{2}} \frac{1}{64 \pi^{2}}\left(-\frac{3}{30}\right)\left(q^{2}\right)^{2} \ln \left(-\frac{q^{2}}{\mu^{2}}\right)=-\frac{m m^{\prime} \delta \pi^{2}}{160 f^{8}}\left(q^{2}\right)^{2} \ln \left(-\frac{q^{2}}{\mu^{2}}\right),
$$

where we have used $\tau \equiv f^{4} / 4 \pi^{2}$ and replaced $\bar{u}\left(p_{3}\right) \gamma^{0}\left(\left(p_{1}+p_{3}\right)^{0} / 2\right) u\left(p_{1}\right)$ and $\bar{u}\left(p_{4}\right) \gamma^{0}\left(\left(p_{2}+\right.\right.$ $\left.\left.p_{4}\right)^{0} / 2\right) u\left(p_{2}\right)$ by the matter net masses $m$ and $m^{\prime}$, respectively.

To obtain the potential, let us now evaluate the Fourier transform:

$$
\begin{aligned}
v(r) & \equiv \int \frac{d^{3} q}{(2 \pi)^{3}} e^{i \boldsymbol{q} \boldsymbol{r}}\left(\boldsymbol{q}^{2}\right)^{2} \ln \frac{\boldsymbol{q}^{2}}{\mu^{2}} \\
& =\frac{1}{\pi^{2}} \int_{0}^{\infty} q^{2} d q \frac{e^{i q r}-e^{-i q r}}{i q r} q^{4} \ln \frac{q^{2}}{\mu^{2}} \\
& =\frac{1}{\pi^{2} r} \operatorname{Im} \int_{0}^{\infty} d q e^{i q r} q^{5} \ln (q / \mu) .
\end{aligned}
$$

This is divergent in the ultraviolet region $q \rightarrow \infty$. However, the amplitude $\mathcal{M} \sim$ $q^{4} \ln q^{2}$ is reliable only in the infrared region and the true amplitude should be suppressed well in the ultraviolet region. Supposing so, we put here a suppression factor $e^{-\epsilon q}$, or making a replacement $r \rightarrow r+i \epsilon$, in the integrand and so understand that the obtained result is reliable only for large $r$.

Then, we can rotate the integration contour $0 \leq q \leq \infty$ counterclockwise into that along the imaginary axis, $0 \leq \kappa \leq \infty$ with $q=i \kappa$ since the contribution from the quarter circle at infinity vanishes thanks to the factor $e^{i q r-\epsilon q}$. Putting $\epsilon=0$, we then obtain

$$
v(r)=\frac{1}{\pi^{2} r} \operatorname{Im} \int_{0}^{\infty} d \kappa e^{-\kappa r} \kappa^{5} i^{6} \ln (i \kappa / \mu)=\frac{1}{\pi^{2} r} \int_{0}^{\infty} d \kappa e^{-\kappa r} \kappa^{5}\left(-\frac{\pi}{2}\right)=-\frac{60}{\pi} \frac{1}{r^{7}} .
$$

Thus the desired potential is found to be

$$
V(r)=-\left(-\frac{m m^{\prime} \delta \pi^{2}}{160 f^{8}}\right)\left(-\frac{60}{\pi}\right) \frac{1}{r^{7}}=-\frac{3 \pi \delta}{8 f^{8}} \frac{m m^{\prime}}{r^{7}},
$$

where the overall negative sign has been put since the amplitude $\mathcal{M}$ corresponds to the effective action which has opposite sign to the potential energy. 


\section{Calculation of the cross sections $\sigma_{a}$ and $\sigma_{b}$}

The amplitude for the production process (a) in Fig. 2 of the level $n$ KK graviton $h_{\mu \nu}^{(n)}$ is calculated using the gravity interaction term (3.5) and is given by

$$
\mathcal{M}_{n}=-\kappa_{n} \varepsilon^{* \mu \nu}(\boldsymbol{k}, \lambda) \bar{v}\left(\boldsymbol{p}_{2}, s_{2}\right) \gamma_{\nu} \frac{\left(p_{1}-p_{2}\right)_{\mu}}{2} u\left(\boldsymbol{p}_{1}, s_{1}\right)
$$

where $\kappa_{n} \equiv \kappa \exp \left(-\frac{1}{2}\left(\frac{n}{R}\right)^{2} \frac{M^{2}}{f^{4}}\right)$ is the $n$-th KK graviton coupling strength suppressed by the brane fluctuation effect, and $\varepsilon_{\mu \nu}$ is the polarization tensor of the massive KK graviton. The initial-spin averaged cross section in the center-of-mass frame is given by the standard formula:

$$
\sigma_{a}=\sum_{n} 2 \pi \delta\left(\sqrt{s}-k_{0}\right) \frac{\frac{1}{4} \sum_{s_{1}, s_{2}, \lambda}\left|\mathcal{M}_{n}\right|^{2}}{4 s \sqrt{s-4 m_{e}^{2}}}
$$

where $s=\left(p_{1}+p_{2}\right)^{2}$, and $\sum_{n}$ denotes the summation over the level number $n$ of the final KK graviton. Henceforth we will set the small electron mass $m_{e}$ equal to zero. The spin sum for the final massive KK graviton is done by using

$$
\sum_{\lambda=-2}^{2} \varepsilon^{* \mu \nu}(\boldsymbol{k}, \lambda) \varepsilon^{\rho \sigma}(\boldsymbol{k}, \lambda)=\frac{1}{2}\left(\eta^{\mu \rho} \eta^{\nu \sigma}+\eta^{\mu \sigma} \eta^{\nu \rho}-\frac{2}{3} \eta^{\mu \nu} \eta^{\rho \sigma}+\cdots\right),
$$

where the ellipses denote the terms containing $k^{\alpha} k^{\beta} / m_{n}^{2}(\alpha, \beta=\mu, \nu, \rho, \sigma)$, which all do not contribute to the cross section because of the energy-momentum tensor conservation. The third term $(2 / 3) \eta^{\mu \nu} \eta^{\rho \sigma}$ does not contribute either since the energymomentum tensor is traceless when $m_{e}=0$. Thus we obtain

$$
\frac{1}{4} \sum_{s_{1}, s_{2}, \lambda}\left|\mathcal{M}_{n}\right|^{2}=\frac{\kappa_{n}^{2}}{8} s^{2}
$$

The mass of the level $n$ KK graviton is given by $m_{n}^{2}=n^{2} / R^{2}$ which equals $k_{0}^{2}$ in the center-of-mass frame, and so the summation $\sum_{n}$ over $n$ can be replaced by the integral $\int R^{\delta} d^{\delta} k_{0}=R^{\delta} \Omega_{\delta} \int k_{0}^{\delta-1} d k_{0}$ with the solid angle $\Omega_{\delta}=2 \pi^{\delta / 2} / \Gamma\left(\frac{\delta}{2}\right)$ in $\delta$-dimensions. Using also the relation $\kappa^{-2}=(2 \pi R)^{\delta} M^{\delta+2}$, we finally get

$$
\sigma_{a}=\frac{\pi^{1-\frac{\delta}{2}}}{2^{\delta+3} \Gamma\left(\frac{\delta}{2}\right)} \frac{s^{\delta / 2}}{M^{\delta+2}} e^{-s \frac{M^{2}}{f^{4}}}
$$

The amplitude for the NG boson pair production process (b) in Fig. 2 is calculated using the interaction term (3.4) and is given by

$$
\mathcal{M}_{\phi \phi}=\frac{1}{2 \tau}\left(-k_{1}^{\mu} k_{2}^{\nu}-k_{1}^{\nu} k_{2}^{\mu}\right) \bar{v}\left(\boldsymbol{p}_{2}, s_{2}\right) \gamma_{\nu} \frac{\left(p_{1}-p_{2}\right)_{\mu}}{2} u\left(\boldsymbol{p}_{1}, s_{1}\right)
$$


The initial-spin averaged cross section in the center-of-mass frame $\left(\boldsymbol{p}_{1}=-\boldsymbol{p}_{2} \equiv\right.$ $\left.\boldsymbol{p}, \boldsymbol{k}_{1}=-\boldsymbol{k}_{2} \equiv \boldsymbol{k}\right)$ is given by the standard formula:

$$
\sigma_{b}=\frac{1}{2} \delta \int \frac{d \Omega}{64 \pi^{2}} \frac{|\boldsymbol{k}|}{s|\boldsymbol{p}|} \frac{1}{4} \sum_{s_{1}, s_{2}}\left|\mathcal{M}_{\phi \phi}\right|^{2},
$$

where the factor $1 / 2$ in front is put because the final two particles are of the same kind, and the factor $\delta$ comes from the sum over the index $m$ of the NG bosons $\phi^{m}$. Since we are neglecting $m_{e}$ presently, we have $|\boldsymbol{k}|=|\boldsymbol{p}|$, and

$$
\frac{1}{4} \sum_{s_{1}, s_{2}}\left|\mathcal{M}_{\phi \phi}\right|^{2}=\frac{1}{128 \tau^{2}} s^{4} \cos ^{2} \theta\left(1-\cos ^{2} \theta\right),
$$

with $\cos \theta \equiv \boldsymbol{p} \cdot \boldsymbol{k} /|\boldsymbol{p}||\boldsymbol{k}|$. Putting $\tau=f^{4} / 4 \pi^{2}$, we find the cross section to be

$$
\sigma_{b}=\frac{\delta \pi^{3}}{1920} \frac{s^{3}}{f^{8}} .
$$




\section{References}

[1] I. Antoniadis, Phys. Lett. 246B (1990) 377; J.D. Lykken, Phys. Rev. D54 (1996) 3693; N. Arkani-Hamed, S. Dimopoulos and G. Dvali, Phys. Lett. 429B (1998) 263; I. Antoniadis, N. Arkani-Hamed, S. Dimopoulos and G. Dvali, Phys. Lett.

436B (1998) 257; N. Arkani-Hamed, S. Dimopoulos and J. March-Russell, hepth/9809124.

[2] K.R. Dienes, E. Dudas and T. Gherghetta, Phys. Lett. 436B (1998) 55; Nucl. Phys. B537 (1999) 47; S. Abel and S. King, Phys. Rev. D59 (1999) 095010; N. Arkani-Hamed and S. Dimopoulos, hep-ph/9811353; N. Arkani-Hamed and M. Schmaltz, Phys. Rev. D61 (2000) 033005; H.-C. Cheng, Phys. Rev. D60 (1999) 075015; K. Yoshioka, Mod. Phys. Lett. A15 (2000) 29.

[3] K.R. Dienes, E. Dudas and T. Gherghetta, Nucl. Phys. B557 (1999) 25; N. Arkani-Hamed, S. Dimopoulos, G. Dvali and J. March-Russell, hepph/9811448; A.E. Faraggi and M. Pospelov, Phys. Lett. 458B (1999) 237; G. Dvali and A.Yu. Smirnov, Nucl. Phys. B563 (1999) 63; R.N. Mohapatra, S. Nandi and A. Prez-Lorenzana, Phys. Lett. 466B (1999) 115.

[4] I. Antoniadis, C. Muñoz and M. Quirós, Nucl. Phys. B397 (1993) 515; E.A. Mirabelli and M.E. Peskin, Phys. Rev. D58 (1998) 065002; I. Antoniadis, S. Dimopoulos, A. Pomarol and M. Quiros, Nucl. Phys. B544 (1999) 503.

[5] Z. Berezhiani and G. Dvali, Phys. Lett. 450B (1999) 24; Z. Kakushadze, Nucl. Phys. B551 (1999) 549; H.-C. Cheng and K.T. Matchev, Nucl. Phys. B563 (1999) 21; Y. Sakamura, hep-ph/9909454.

[6] N. Arkani-Hamed, S. Dimopoulos and G. Dvali, Phys. Rev. D59 (1999) 086004; M. Maggiore and A. Riotto, Nucl. Phys. B548 (1999) 427; N. Kaloper and A. Linde, Phys. Rev. D59 (1999) 101303; N. Arkani-Hamed, S. Dimopoulos, N. Kaloper and J. March-Russell, hep-ph/9903224; hep-ph/9903239; A. Riotto, hep-ph/9904485.

[7] G. Shiu and S.H.H. Tye, Phys. Rev. D58 (1998) 106007; Z. Kakushadze and S.H.H. Tye, Nucl. Phys. B548 (1999) 180; I. Antoniadis and C. Bachas, Phys. Lett. 450B (1999) 83; Z. Kakushadze, Nucl. Phys. B552 (1999) 3; G. Shiu, 
R. Shrock and S.H.H. Tye, Phys. Lett. 458B (1999) 274; E.E. Flanagan, S.H.H. Tye and I. Wasserman, hep-ph/9909373.

[8] M. Bando, T. Kugo, T. Noguchi and K. Yoshioka, Phys. Rev. Lett. 83 (1999) 3601 (hep-ph/9906549).

[9] R. Sundrum, Phys. Rev. D59 (1999) 085009.

[10] J. Polchinski, String Theory, vol. 1, 2 (Cambridge Univ. Press, Cambridge, 1998).

[11] For example, G.F. Giudice, R. Rattazzi and J.D. Wells, Nucl. Phys. B544 (1999) 3; S. Nussinov and R. Shrock, Phys. Rev. D59 (1999) 105002; E.A. Mirabelli, M. Perelstein and M.E. Peskin, Phys. Rev. Lett. 82 (1999) 2236; T. Han, J.D. Lykken and R.-J. Zhang, Phys. Rev. D59 (1999) 105006; J.L. Hewett, Phys. Rev. Lett. 82 (1999) 4765; P. Mathews, et al., Phys. Lett. 450B (1999) 343; ibid. 455B (1999) 115; hep-ph/9904232; Phys. Lett. 461B (1999) 196; T.G. Rizzo, Phys. Rev. D59 (1999) 115010; ibid. D60 (1999) 075001; ibid. D60 (1999) 115010; hep-ph/9910255; K. Cheung and W.-Y. Keung, Phys. Rev. D60 (1999) 112003; K. Cheung, Phys. Rev. D61 (2000) 015005; D. Atwood, S. BarShalom and A. Soni, hep-ph/9903538; hep-ph/9906400; hep-ph/9909392; hepph/9911231.

[12] N. Arkani-Hamed, S. Dimopoulos and G. Dvali, Phys. Rev. D59 (1999) 086004.

[13] M.S. Chanowitz and M.K. Gaillard, Nucl. Phys. B261 (1985) 379; K.-I. Aoki, talk presented at meeting on "Physics at TeV Energy Scale", Tsukuba, May, 1987, (QCD161:M376:1987).

[14] I. Antoniadis and K. Benakli, Phys. Lett. 326B (1994) 69; J. Hisano and N. Okada, hep-ph/9909555.

[15] Particle Data Group, C. Caso et al., Eur. Phys. J. C3 (1998) 1.

[16] G.B. Gelmini, S. Nussinov and T. Yanagida, Nucl. Phys. B219 (1983) 31; J.E. Moody and F. Wilczek, Phys. Rev. D30 (1984) 130; T.R. Taylor and G. Veneziano, Phys. Lett. 213B (1988) 450; S. Dimopoulos and G.F. Giudice, Phys. Lett. 379B (1996) 105; I. Antoniadis, S. Dimopoulos and G. Dvali, Nucl. Phys. B516 (1998) 70. 
[17] J.C. Long, H.W. Chan and J.C. Price, Nucl. Phys. B539 (1999) 23.

[18] G. Feinberg, J. Sucher and C.-K. Au, Phys. Rep. 180 (1989) 83.

[19] A. Kehagias and K. Sfetsos, Phys. Lett. 472B (2000) 39; E.G. Floratos and G.K. Leontaris, Phys. Lett. 465B (1999) 95.

[20] S. Cullen and M. Perelstein, Phys. Rev. Lett. 83 (1999) 268; V. Barger, T. Han, C. Kao and R.-J. Zhang, Phys. Lett. 461B (1999) 34.

[21] C.J. Horowitz and J. Pantaleone, Phys. Lett. 319B (1993) 186; F. Ferrer and M. Nowakowski, Phys. Rev. D59 (1999) 075009.

[22] J.K. Hoskins, R.D. Newman, R. Spero and J. Schultz, Phys. Rev. D32 (1985) 3084; V.P. Mitrofanov and O.I. Ponomareva, Sov. Phys. JETP 67 (1988) 1963.

[23] S.K. Lamoreaux, Phys. Rev. Lett. 78 (1997) 5.

[24] For a review, G.G. Raffelt, Phys. Rep. 198 (1990) 1; hep-ph/9903472.

[25] J. Ellis and K.A. Olive, Nucl. Phys. B223 (1983) 252; N. Iwamoto, Phys. Rev. Lett. 53 (1984) 1198; R. Mayle et al., Phys. Lett. 203B (1988) 188; ibid. 219B (1989) 515; G. Raffelt and D. Seckel, Phys. Rev. Lett. 60 (1988) 1793.

[26] M.S. Turner, Phys. Rev. Lett. 60 (1988) 1797; A. Burrows, M.T. Ressell and M.S. Turner, Phys. Rev. D42 (1990) 3297.

[27] J. Engel, D. Seckel and A.C. Hayes, Phys. Rev. Lett. 65 (1990) 960.

[28] K. Hirata, et al., Phys. Rev. Lett. 58 (1987) 1490.

[29] R.M. Bionta, et al., Phys. Rev. Lett. 58 (1987) 1494.

[30] M. Bando, T. Kugo and K. Yamawaki, Phys. Rep. 164 (1988) 217, and references therein. 